\title{
Review Article \\ Nanosphere Lithography: A Powerful Method for the Controlled Manufacturing of Nanomaterials
}

\author{
Pierre Colson, ${ }^{1}$ Catherine Henrist, ${ }^{1,2}$ and Rudi Cloots ${ }^{1,2}$ \\ ${ }^{1}$ University of Liege, Chemistry Department, Group of Research in Energy and Environment from Materials (GREENMAT), \\ Allée de la Chimie 3 (Building B6), 4000 Liege (Sart Tilman), Belgium \\ ${ }^{2}$ University of Liege, Center for Applied Technology in Microscopy (CAT $)$ ), Allée de la Chimie 3 (Building B6), \\ 4000 Liege (Sart Tilman), Belgium \\ Correspondence should be addressed to Pierre Colson; pierre.colson@ulg.ac.be and Rudi Cloots; rcloots@ulg.ac.be
}

Received 10 July 2013; Accepted 26 August 2013

Academic Editor: Tifeng Jiao

Copyright ( $\odot 2013$ Pierre Colson et al. This is an open access article distributed under the Creative Commons Attribution License, which permits unrestricted use, distribution, and reproduction in any medium, provided the original work is properly cited.

The never-ending race towards miniaturization of devices induced an intense research in the manufacturing processes of the components of those devices. However, the complexity of the process combined with high equipment costs makes the conventional lithographic techniques unfavorable for many researchers. Through years, nanosphere lithography (NSL) attracted growing interest due to its compatibility with wafer-scale processes as well as its potential to manufacture a wide variety of homogeneous one-, two-, or three-dimensional nanostructures. This method combines the advantages of both top-down and bottom-up approaches and is based on a two-step process: (1) the preparation of a colloidal crystal mask (CCM) made of nanospheres and (2) the deposition of the desired material through the mask. The mask is then removed and the layer keeps the ordered patterning of the mask interstices. Many groups have been working to improve the quality of the CCMs. Throughout this review, we compare the major deposition techniques to manufacture the CCMs (focusing on 2D polystyrene nanospheres lattices), with respect to their advantages and drawbacks. In traditional NSL, the pattern is usually limited to triangular structures. However, new strategies have been developed to build up more complex architectures and will also be discussed.

\section{Introduction}

More than 50 years have passed since the famous lecture "There's plenty of room at the bottom" by Feynman [1]. In his talk, Feynman explored apparently simple and elegant possibilities of working at the atomic scale-possibilities that would have startling results. He touched upon ideas such as miniature writing, seeing and moving atoms, the prospect of designing molecules one atom at a time, and the challenges involved in developing miniature machines. Devices operating on this scale are expected to provide a number of breakthrough applications like more powerful computers and increased data storage due to more efficient and smaller components. Applications are also expected in many fields such as solar cells, (molecular) electronics, and biosensors.
To cite Feynman, the question is now, "How do we write small?"

There are two well-known major routes for nanofabrication, called the "top-down" and the "bottom-up" approaches [2-8].

1.1. Top-Down. In the top-down approach, thin films or bulk materials are scaled down to create nanodevices. This can be done by using various techniques such as precision engineering and lithography and has been developed and refined by the semiconductor industry over the past 30 years. Indeed, the never-ending race towards miniaturization of devices (mobile, camera, etc.) induced an intense research in the manufacturing processes of the basic components of these devices. Subtractive and additive transfers lead to different nanoarchitectured materials. 
The lithographic tools are classified in two families: "parallel writing" (or replication) methods (e.g., optical lithography) and "sequential writing" (or patterning) methods (e.g., focused-electron beam lithography).

In parallel writing, the whole pattern is made simultaneously using a mask, which dictates the features to be reproduced. It is easy to understand that sequential lithography is slower as the pattern is written point by point on the resist surface and is therefore more expensive.

Optical lithographic processes are limited in final resolution by the wavelength of the light used to expose the photoresist [10]. The use of short wavelengths and reduction optics between the mask and substrate has allowed a reduction in features size. Extending these processes to wavelengths in deep UV and soft X-ray brings increasing technical difficulty. Alternative techniques, such as electron beam lithography, have been developed to overcome the limitations of optical light. While optical lithography is mainly intended for patterns of a few micrometers to a few 100 microns, the electron beam lithography allows for its part to achieve submicron resolutions far. Under ideal conditions, it is possible to reach a sub-10 $\mathrm{nm}$ resolution.

Each of these conventional fabrication tools, except for soft X-ray lithography, is commercially available (though expensive). These techniques are highly developed and optimized for semiconductor fabrication. The costs of purchasing, installing, and maintaining the tools they require limit their application in areas other than microelectronics. These tools are also often incompatible with nonstandard problems in fabrication (e.g., coatings on nonplanar surfaces).

Over the years, many lithographic and patterning techniques have been proposed to overcome these disadvantages. The concept of soft lithography was introduced in 1988 by Whiteside's group at Harvard [11]. It refers to a set of methods $[4,12,13]$ (microcontact printing, replica molding, microtransfer molding, etc.) for generating or replicating structures by using a patterned elastomer (e.g., polydimethylsiloxane (PDMS)) as a mask, stamp, or mold. These methods can be carried out by hand in an ordinary laboratory. Conventional photolithography must take place in clean-room facility devoid of dust and dirt; if a piece of dust lands on the mask, it will create an unwanted spot on the pattern. As a result, the device being fabricated may fail. Soft lithography is generally more forgiving because the PDMS stamp is elastic. If a piece of dust gets trapped between the stamp and the surface, the stamp will compress over the top of the particle but maintain contact with the surface. Thus, the pattern will be reproduced correctly except for where the contaminant is trapped.

Moreover, soft lithography can produce nanostructures in a wide range of materials, including the complex organic molecules needed for biological studies [14]. The technique can print or mold pattern on curved or planar surfaces, but the technology is not ideal for making the structures required for complex nanoelectronics. Currently all integrated circuits consist of stacked layers of different materials. Deformations of the soft PDMS stamp can produce small errors in the replicated pattern and a misalignment of the pattern with any underlying patterns previously fabricated.
Therefore, soft lithography is not well suited for fabricating structures with multiple layers that must stack precisely on top of one another.

Researchers have found ways, however, to correct this shortcoming - at least in part-by employing a rigid stamp instead of an elastic one.

In a technique called step and flash imprint lithography [13], photolithography is used to etch a pattern into a quartz plate, yielding a rigid bas-relief master. The master is then pressed against a thin film of liquid polymer, which fills masters recesses. Exposition to ultraviolet light solidifies the polymer to create the desired replica. A related technique called nanoimprint lithography [15] also employs a rigid master but uses a film of polymer that has been heated to a temperature near its melting point to facilitate the embossing process.

Scanning probe techniques [16] are also potential tools for nanofabrication. Depending on the sharpness of the pen, the resolution of writing can vary from macroscopic feature sizes down to the atomic sale. These scanning probe techniques are based on the changes undergone by species present on the surface of a substrate.

In practice, such changes can be induced by mechanical, electrical, magnetic, or chemical means. One of the most striking examples of nanoscale fabrication by scanning probe lithography has been the precise positioning of atoms with an STM tip [17]. Manipulations of atoms or particles by scanning probe lithography techniques will be rather classified in the bottom-up approach. These tools seem well suited for applications in research but will require substantial development (simultaneous writing patterns with multiple probes [18]) before they can be used for patterning large areas in manufacturing. The characteristics of all these lithography techniques are summarized in Table 1.

Many reviews are focused on the evolution of these new "unconventional" approaches to nanofabrication $[13,19,20]$.

1.2. Bottom-Up. Bottom-up approach uses small and simple building blocks (atoms, molecules, nanoparticles, etc.) that will self-assemble into larger, more complex structures. Compared with top-down route, this is more like a chemical or biological approach and it has the potential to make complex 3D structures cheaply and in large quantities. These methods can easily make the smallest nanostructures-easily reaching sub-10 nm dimensions - and do so inexpensively.

Self-assembly (although the distinction is not always made in the literature, the terms self-assembly and selforganization should be used, resp., to describe structures formed close-to-equilibrium and far-from-equilibrium dissipative processes involving energy/matter flow. In some cases, the words are used interchangeably and the confusion is still frequent in the literature.) is defined as the spontaneous (without the assistance of external forces or spatial constraints) organization of two (or more) components into larger aggregates using covalent and/or noncovalent bonds [24].

Materials fabricated using this approach include selfassembled monolayers [25] or nanostructures that selfassemble from block copolymers [23, 26-28] and that can 
TABLE 1: Characteristics of most popular lithographic techniques $[7,13,19,20]$.

\begin{tabular}{|c|c|c|c|c|c|c|}
\hline Technique & Minimum feature size & Pattern & Area & Cost & Time & Use \\
\hline Deep UV L. [21] & $\cong 50-100 \mathrm{~nm}$ & PW & Large & High & Short & Industry \\
\hline Immersion deep UV L. [22] & $\cong 30 \mathrm{~nm}$ & PW & Large & High & Short & R \& D \\
\hline Extreme UV L. [23] & $<50 \mathrm{~nm}$ & PW & Large & High & Short & $\mathrm{R} \& \mathrm{D}$ \\
\hline X-Ray L. & $\cong 20 \mathrm{~nm}$ & PW & Large & High & Short & $\mathrm{R} \& \mathrm{D}$ \\
\hline Electron Beam L. & $\cong \mathrm{nm}$ & SW & Small & High & Long & $\mathrm{R} \& \mathrm{D}$ (industry) \\
\hline Soft L. [11] & $\cong 10 \mathrm{~nm}$ & PW & Large & Low & Short & $\mathrm{R} \& \mathrm{D}$ (industry) \\
\hline Scanning Probe L. [16] & $<1 \mathrm{~nm}$ & SW & Small & High & Long & R \& D \\
\hline
\end{tabular}

L.: lithography; PW: parallel writing; SW: sequential writing.

be used as templates for the preparation of metallic or semiconducting nanostructures [29]. The main advantage of this technique is that nanoscale features can be achieved. Block-copolymer lithography is sometimes used together with a lithography prepatterned substrate for further specific assembly of copolymers [23].

Recent advances in the fabrication of functional nanostructures using self-assembly include self-assembled [30] magnetic nanoparticles [31]. Crystalline areas of magnetic nanocrystals can store large amount of information. However, an annealing step is usually necessary to enhance the magnetic properties and may give rise to coalescence of the nanoparticles [32]. Nontemplated self-assembly, while being attractive for its minimalist use of materials and energy (compared to conventional lithography), is not widely used for nanofabrication. Indeed, self-assembly is prone to producing defects, and the perfect periodicity of self-assembled structures from nanoscale components is generally limited to micrometer-sized areas [13]. Moreover, precise spatial positioning and nanostructure shape control are difficult to implement.

1.3. Top-Down, Bottom-Up, or Both? Considering the previously described synthesis routes (Sections 1.1 and 1.2), the following conclusions can be drawn. The complexity of the preparation process combined with high initial equipment costs makes the conventional lithographic techniques unfavorable for many researchers.

Most of the unconventional lithographic techniques developed to date, however, require the assistance of conventional lithographic techniques, such as photolithography, to design and manufacture the masks or masters. Soft lithography techniques, although they present many advantages, do require the production of a single mask (which will be used several times) by conventional lithographic techniques. Scanning probe lithography techniques are of course too slow for mass production. Bottom-up techniques, although they easily reach sub-10 nanometers, may present some disadvantages. Self-assembly has its own problem of regularity and repeatability. Indeed, control of the density and shape of the nanostructures is difficult to achieve, which is rather annoying especially in case of magnetic data storage.

By the way, the dimensions that can now be controlled by either approach (Figure 1) are of similar order, and this

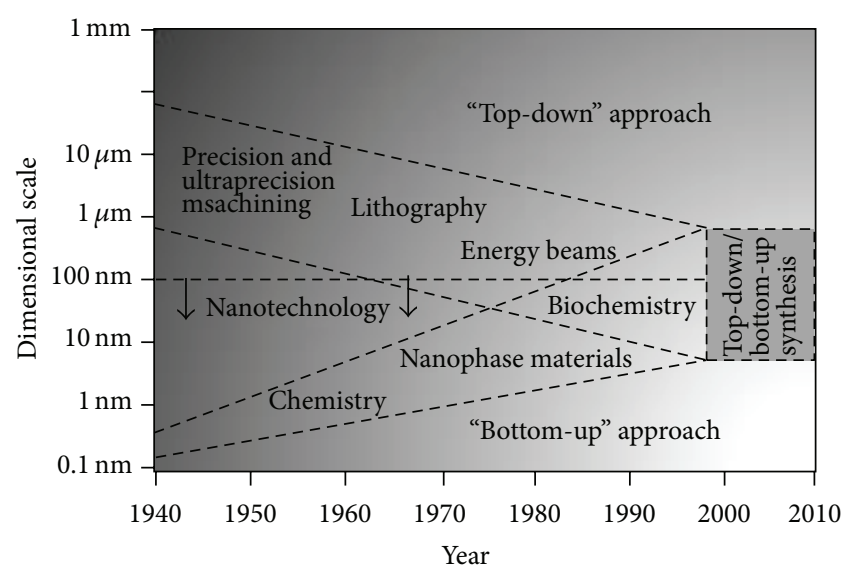

FIGURE 1: The convergence of top-down and bottom-up approaches [9].

leads to exciting new hybrid methods including nanosphere lithography towards which our choice was directed.

\section{Nanosphere Lithography}

Nanosphere lithography (NSL) is a promising inexpensive fabrication tool for producing regular and homogenous arrays of nanoparticles with different sizes. This method combines the advantages of both top-down and bottom-up approaches.

The process is divided into two steps (Figure 2), the first of which is mask preparation.

The flat substrate is coated with a suspension containing monodisperse spherical colloids (e.g., polystyrene) after a chemical treatment to enhance its hydrophilic character. Upon drying, a hexagonal-close-packed (HCP) monolayer or bilayer, called a colloidal crystal mask [33] (CCM), is formed.

This mask is then used to selectively pattern the substrate thanks to the deposition of the material of interest through the interstices of the ordered beads.

The subsequent removal of the mask (lift-off) by sonication in an adequate solvent or by stripping leaves an array of ordered nanodots on the surface of the substrate. An annealing step is sometimes necessary to crystallize the sample or/and induce a crystallographic phase change. 


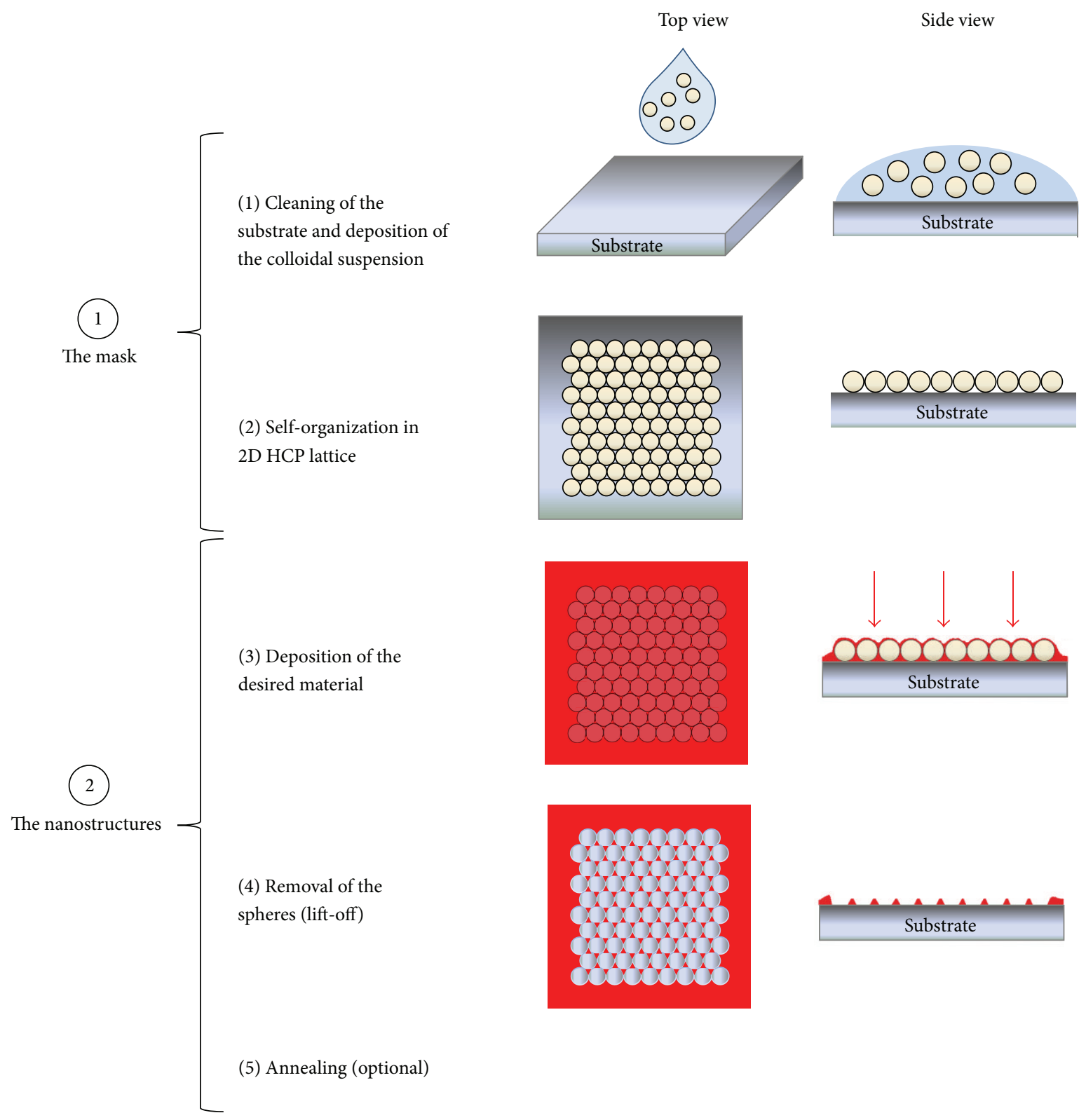

FIGURE 2: Nanosphere lithography process (NSL).

The NSL is often considered as a hybrid between the bottom-up approach (due to the self-organization of the colloidal spheres in a HCP lattice) and the top-down approach (due to the obtention of dots/structured layers such as in a conventional lithography technique).

Nanosphere lithography is also known as colloidal lithography $[34,35]$ or natural lithography.

Fischer and Zingsheim [36] are the first to report, in 1981, the formation of an ordered monolayer on a glass plate. They simply deposited a suspension of colloidal spheres with a diameter of $312 \mathrm{~nm}$ and allowed it to evaporate. They obtained small-area particle monolayers. The term of "naturally" assembled polystyrene (PS) latex nanospheres was then introduced. However, the focus of their work was the replication of submicroscopic patterns using visible light and not fabrication of lithographic colloidal masks.

A year later, Deckman and Dunsmuir [37] extend the scope of the approach of Fischer showing that a monolayer of nanospheres can be used both as a "deposit material" or as a lithographic mask. The deposition is either random or with ordered arrays over the entire surface of a macroscopic substrate [38]. The coating procedure developed to produce microcrystalline arrays is based on a spin coating process. Several point defects or dislocations are present in the tens of micrometers scale. Because the mask preparation process is obtained by self-assembly phenomenon rather than by photolithography, they named this strategy "natural lithography." 
SL

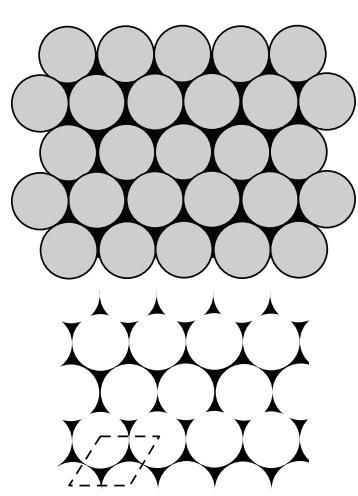

DL

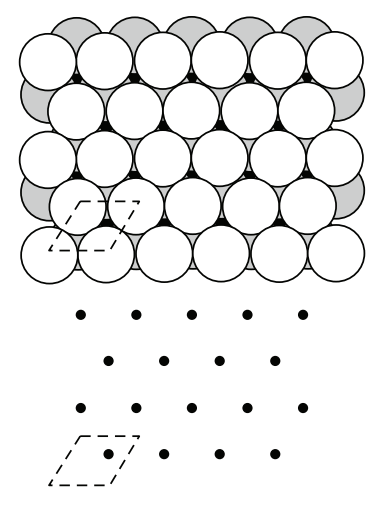

FIGURE 3: Schematic diagrams of single-layer (SL) and double-layer (DL) nanosphere masks and the corresponding periodic particle array PPA surfaces (dotted line = unit cell). "Reprinted with permission from [39]. Copyright (1995) American Vacuum Society."

In the 90 s, the method is renamed by the group of Hulteen and Van Duyne [39] and is still nowadays commonly called "nanosphere lithography". Their work is not only focused on mono- (single) layers (SL) but extends to double layers (DL) which leads to the formation of smaller dots corresponding to the small openings that remain in a closed packed structure (Figure 3).

Van Duyne's group [40, 41] has also intensively investigated the plasmon resonance properties of metallic patterns with the ultimate goal of developing biosensors based on surface enhanced Raman spectroscopy.

Besides, several groups have experimentally and theoretically studied the behavior of colloidal suspensions in order to understand their stability as well as the mechanisms of formation of the mask [42].

Through years, nanosphere lithography attracted growing interest due to its potential to manufacture a wide variety of one-, two-, or three-dimensional nanostructures [34, 43-47]. Figure 4 highlights the increasing interest for NSL since 1995.

2.1. Designing Monolayers. In recent years, many groups have been working on different strategies to continually improve the quality of the crystal masks. The major methods will be briefly described below, focusing only on two-dimensional polystyrene nanospheres lattices.

2.1.1. Self-Assembly during Solvent Evaporation. Evaporation methods are based on solvent evaporation from a droplet of dilute colloidal nanosphere suspension deposited on a substrate. The evaporation of the solvent induces the formation of a meniscus between the particles and therefore attractive capillary forces give rise to the self-assembly of the particles.

Denkov and his team [48] conducted pioneering work on self-ordering of colloidal particles by direct observation of colloids. They found that attractive capillary force and convective transport of the nanospheres are the main factors that dominate the self-assembly process, while the ordering

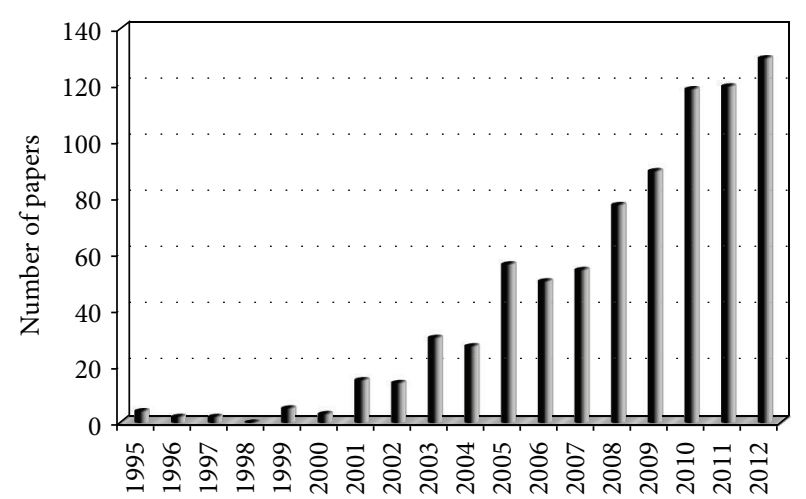

FIGURE 4: Number of papers/year related to NSL, as retrieved by Scifinder Scholar (July 2013).

and quality of the obtained arrays are considerably affected by the rates of solvent evaporation [49].

By controlling the temperature and the humidity of the system, Micheletto et al. [50] prepared colloidal monolayers on a slightly tilted substrate. Since then, other tests were performed successfully $[51,52]$. Low control over the process often yields mixed multilayers structures.

2.1.2. Dip Coating. Based on their work on convective assembly of colloidal nanospheres during solvent evaporation, Nagayama et al. [53, 54] developed a dip-coating procedure for formation of $2 \mathrm{D}$ colloidal templates.

Constant monitoring of the evaporation rate as well as fine-tuning of the withdrawal speed of the substrate are key parameters to achieve large 2D ordered arrays (Figure 5). However, the particles layers are formed on both sides of the substrate, thereby increasing the amount of suspension used.

To overcome this drawback, Nagao et al. [55] engineered a hybrid method between evaporation and dip coating. A drop of colloidal suspension is placed on the substrate, which is thereafter inclined vertically until the suspension is 

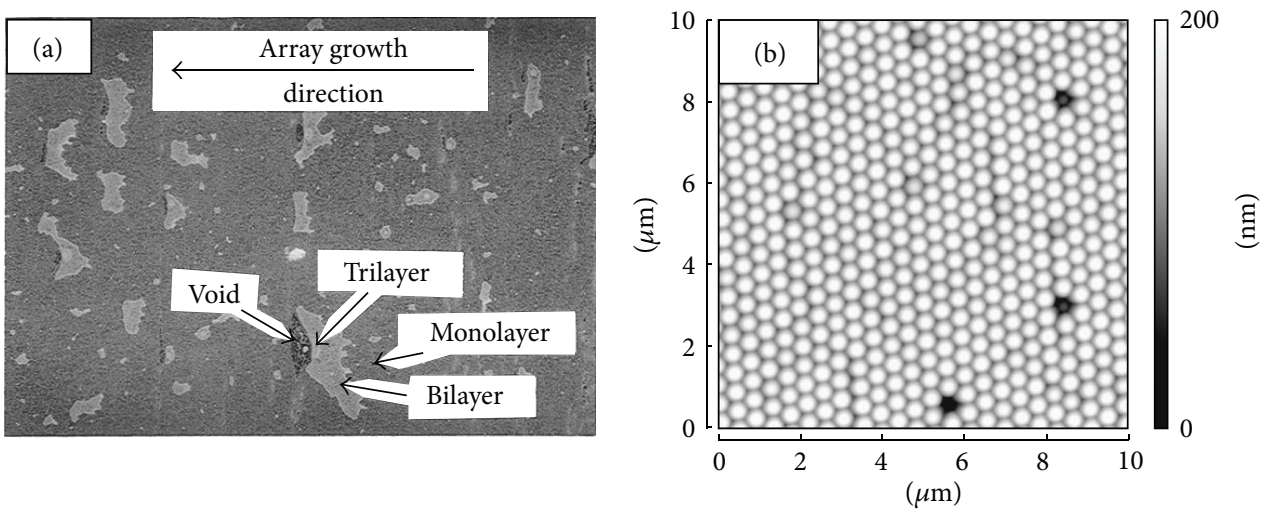

FIGURE 5: (a) SEM micrograph of a PS monolayer (480 nm diameter) containing defects of multilayers and voids. "Reprinted with permission from [53]. Copyright (1996) American Chemical Society.; (b) Tapping-mode atomic force microscopy micrograph of PS monolayer. "Reprinted with permission from [54]. Copyright (2000) American Institute of Physics."

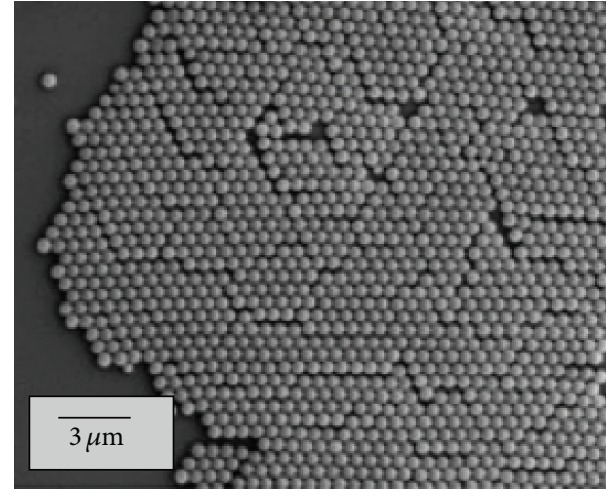

FIGURE 6: SEM micrograph of a PS nanoparticles (520 nm diameter) monolayer. "Reprinted from [55]. Copyright (2007), with permission from Elsevier."

dried. However, many defects are still present in the network (Figure 6). Multilayers can be obtained by repeating the operation.

2.1.3. Spin Coating. The spin coating of a colloidal suspension onto a substrate can accelerate the evaporation of the solvent $[37,39]$. The quality and thickness of colloidal templates are greatly affected by spin speed, size of the nanospheres, wettability, and so forth.

Most of the literature on spin coating of colloidal suspension is experimentally based. Even if recent papers are more detailed, spin coating protocols described in the literature are varied and sometimes imprecise [56-58]. Therefore a major goal for further progress in NSL is the development of experimental protocols to control the ordering of particles on solid substrates and to get large well-ordered structures.

However, the spin coating has a significant potential for mass production and scaling-up because of its rapid implementation and its compatibility with wafer-scale processes.

For those reasons, we studied by an experimental design approach the influence of some spin coating parameters on the degree of ordering of the nanosphere layers (Figure 7) [59]. We highlighted that, to get large hexagonal-closepacked ordered areas, we need to quickly spin coat the suspension at high rotation speed. We also identified adequate spin coating parameters to manufacture large, defectfree areas reaching up to $200 \mu \mathrm{m}^{2}$ (with PS nanospheres of $500 \mathrm{~nm}$ diameter) [59]. These results agree with studies on spin coating of colloidal suspensions $[60,61]$.

As theoretically shown by Kralchevsky and Denkov [49] interparticle capillary forces arise between spherical particles, which are partially immersed in a liquid on a horizontal solid substrate (Figure 8). As the liquid becomes thinner, the liquid surface deformation increases giving rise to increased capillary forces. The next step begins with the motion of more colloids that are driven toward the nucleus and is attributed to a convective flux, which compensates the evaporated solvent in the already ordered array, hence dragging particles suspended in the thicker layers towards the thinner regions. The newcomers remain attached to the domains, pressed by a hydrodynamic pressure and captured by capillary forces.

The onset of ordering process coincides with the moment when the thickness of the liquid layer becomes smaller than the particle diameter [62].

The ordering is therefore highly related to the thickness of the evaporating film. The film thickness has been demonstrated to be inversely proportional to the spin speed $[61,63]$, which corroborates our results. Moreover, Rehg and Higgins [60] and Dushkin et al. [64] both concluded that rapid evaporation rate is better at assembling more uniform monolayers and colloidal crystals. Our results confirm that the first acceleration in the spin coating process is the critical parameter to reach high \% of HCP organization.

Chen et al. [65] recently provided lots of useful information for the efficient and controllable fabrication of largescale and uniform monolayer or multilayer colloid-crystal films with PS nanospheres of various diameters by spincoating technology. They improved the understanding of the relationship between ratios of monolayer coverage areas and spin speed/acceleration. 


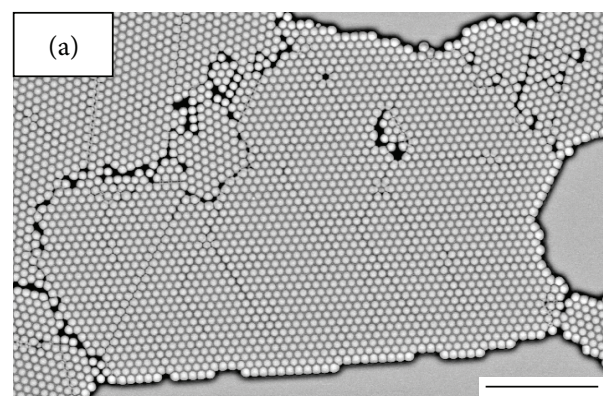

(a)

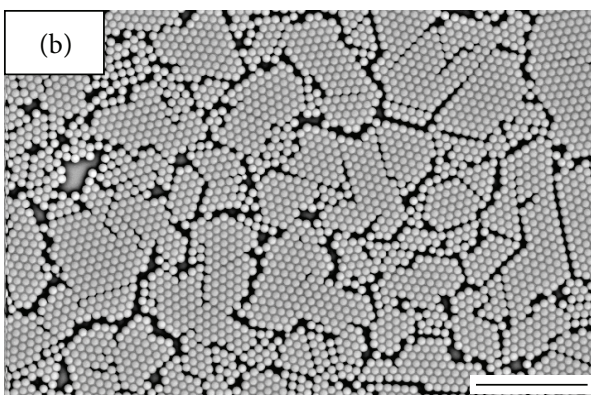

(b)

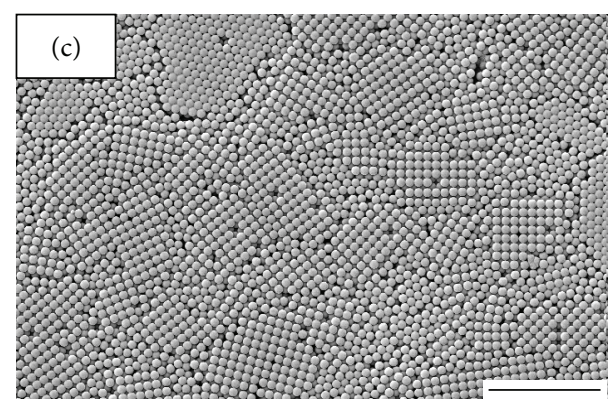

(c)

FIGURE 7: Two- or three-dimensional organization of PS nanospheres (depending on the spin coating parameters). Typical SEM micrographs of organized PS nanospheres showing in (a) large HCP area, (b) small HCP area, and (c) multilayers. Scale bars are $5 \mu \mathrm{m}$. "Reprinted from [59]. Copyright (2011) American Chemical Society."

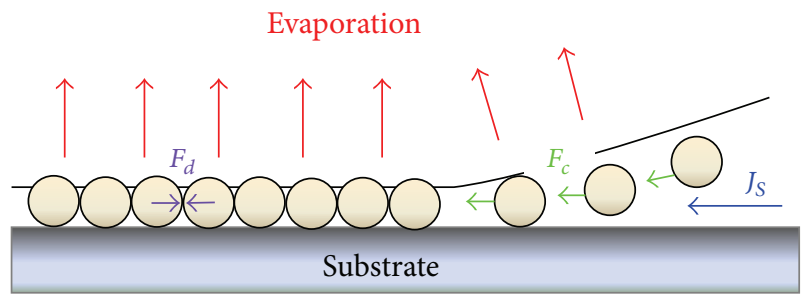

FIGURE 8: Main driving forces in ordering of spherical particles in wetting film on substrate. $F_{C}$ is a capillary force between particles trapped in thin liquid film whose thickness is around the particle diameter. $F_{d}$ is a hydrodynamic force, which drags the immersed particles toward the thinner regions. This force is caused by the hydrodynamic flux $\left(J_{S}\right)$ of the solvent, which compensates the solvent evaporated from thinner regions. "Reprinted from [49]. Copyright (2001), with permission from Elsevier."

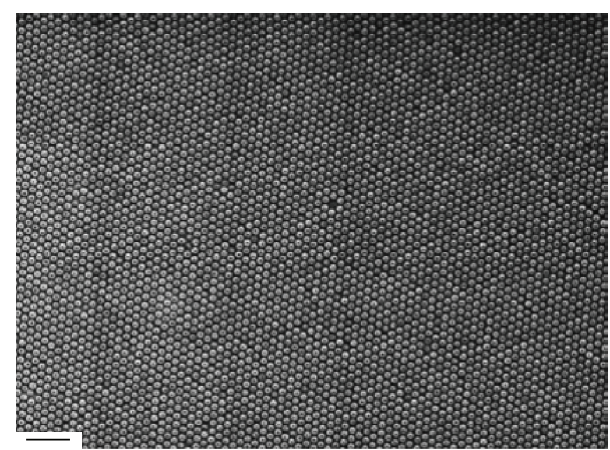

FIGURE 9: SEM micrograph showing large area of self-assembled PS (200 nm diameter) monolayer. The scale bare is $1 \mu \mathrm{m}$. "Reprinted from [70]. Copyright (2009), with permission from Elsevier."

\subsubsection{Self-Assembly at Interface of Two Media}

(i) Air-Liquid Interface

Langmuir-Blodgett Coating. Langmuir-Blodgett coating refers to the preparation and transfer of nanosphere monolayers from liquid-gas interface onto a solid substrate by controlled withdrawal of the substrate. Through use of surfactants [66] or through surface modification of the nanospheres [67] in order to enhance their hydrophobicity, a $2 \mathrm{D}$ crystal film is formed on the liquid surface. The resulting floating monolayer is compressed by a barrier that promotes the assembly of the nanospheres in close-packed lattices. This setup ensures that the pressure remains constant as the substrate is being withdrawn. Macroscopic areas can be patterned with nanospheres.

Controlled Evaporation. The first step of the process (colloidal monolayer formation) is similar to that of the LangmuirBlodgett technique. However, the floating monolayer is left free without action of any barrier to modify its assembly, which greatly simplifies the apparatus. Moreover, instead of being slowly withdrawn from the suspension, the substrate is left immersed during the liquid evaporation $[68,69]$.

By slightly tilting the substrate, large HCP domains (Figure 9) with size greater than $150 \mu \mathrm{m}^{2}$ (PS nanospheres with $200 \mathrm{~nm}$ diameter) were manufactured [70].

\section{(ii) Liquid-Liquid Interface}

Self-assembly may also occur at the interface between two nonmiscible liquids [71]. Thanks to carefully prepared surface conditions, nanospheres assemble at the interface. 


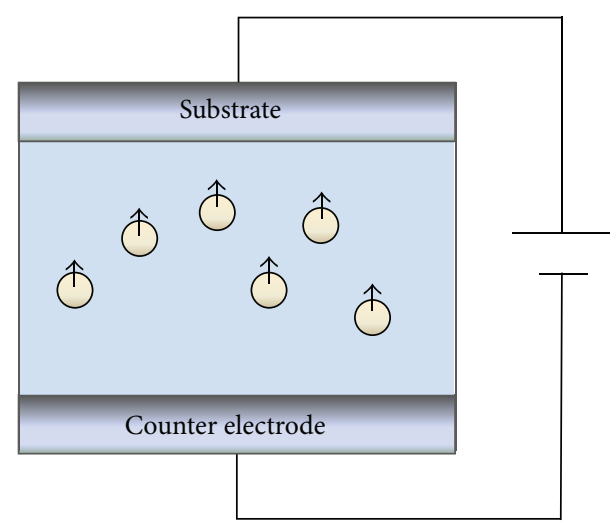

FIGURE 10: Scheme of electrophoretic deposition of colloids.

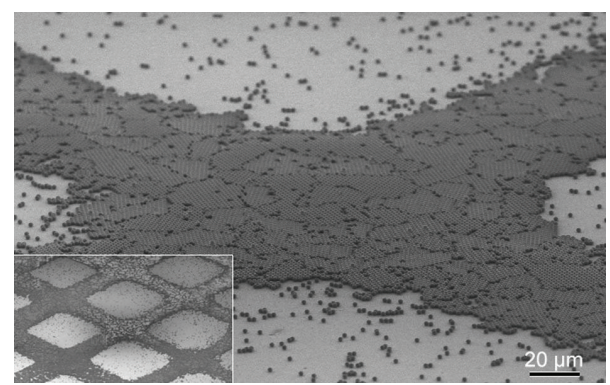

FIGURE 11: SEM micrograph of PS pattern produced by DC electrophoretic deposition in the presence of an UV illumination motif. The inset shows the overall appearance of the pattern. "Reprinted by permission from Macmillan Publishers Ltd. Reference [76], copyright (2000).”

Once they are trapped at the interface, they self-organize into a monolayer. Subsequently, the monolayer is deposited on the substrate by a removal procedure or evaporation of one phase.

2.1.5. Electrophoretic Deposition. In electrophoretic deposition, a colloidal suspension is confined between two electrodes. An applied electric field induces the migration of the particles (Figure 10) and self-assembly occurs at electrode interfaces (Figure 11). This method is therefore limited to conductive substrates (e.g., indium tin oxide (ITO) glass). Two- [72] and three-dimensional [73] colloids crystals have been studied both in a DC field [74-76] or an AC field [77, 78].

The use of AC field allows manipulation of almost any type of particle in any type of media and has the advantage of permitting high field strengths without causing water electrolysis [78].

2.1.6. Prepatterned Substrates. Template-assisted selfassembly of colloids is usually employed to fabricate types of arrangements which are difficult or impossible to fabricate with traditional self-assembly process.

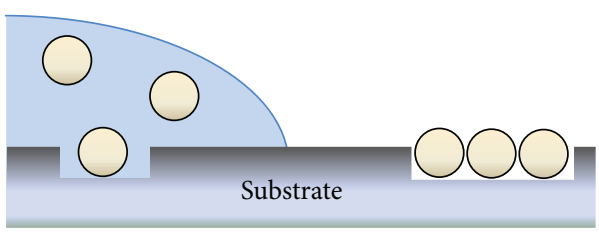

FIGURE 12: Self-assembly on a physically patterned substrate.

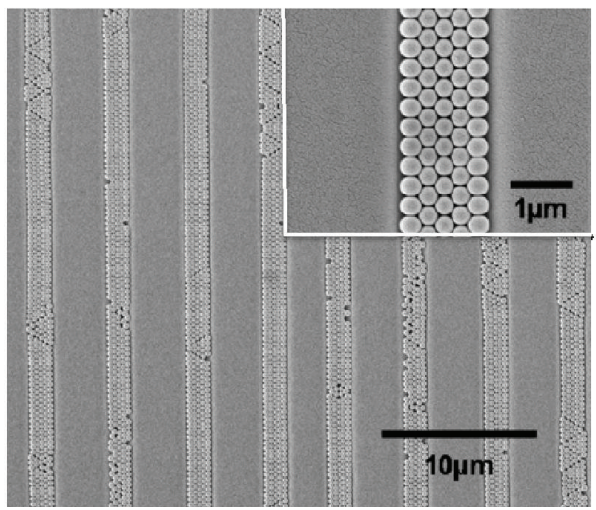

FIGURE 13: SEM micrographs showing large area of polystyrene spheres on patterned photoresist template. Inset shows magnified image of the channel. "Reprinted with permission from [115]. Copyright (2006) American Chemical Society.”

Two fundamentally different strategies are available, namely, the self-assembly on chemically or physically (Figure 12) patterned substrates. Both methods involve an additional preliminary step in the process and may consequently increase the time and the cost of preparation.

Indeed, physically patterned substrates are produced by conventional lithographic techniques (optical lithography [79] and electron beam lithography). By changing the shape of the patterned geometry and the ratio size to particle size (Figures 13 and 14), it is possible to control the packing order of the colloidal crystals [80,81].

On the other side, a selective deposition of the colloids may occur on chemically patterned (Figure 15) substrates [82, 83].

Depending on the nature of the interaction between the particles and the template surface, the assembly process is carried out by different methods (evaporation, spin coating, or electrophoretic deposition (Figure 16)).

\section{Applications of NSL}

Assembling colloidal micro-nanoparticles into 2D ordered arrangements presents a high potential for applications in many different fields.

First, freestanding cross-linked polymer nanoparticle films can be used as filtration membranes to separate small proteins or gold nanoparticles and present the great advantage of a narrow pore size distribution that is never seen in the present polymer membranes [84].

Furthermore, the 2D HCP monolayer can be used as mask or template to generate patterns of functional materials on 

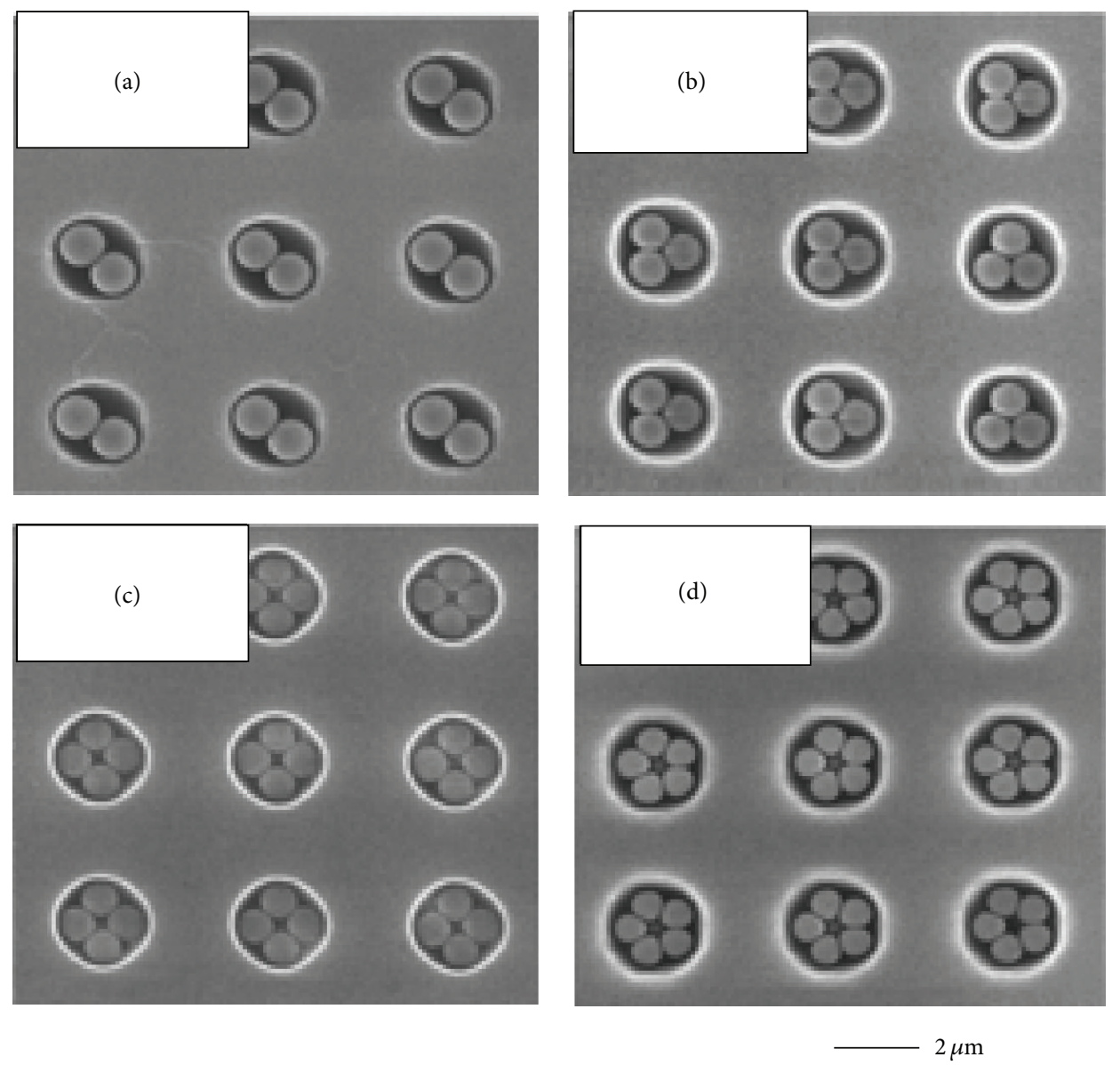

FIGURE 14: SEM micrographs of some typical examples of polygonal aggregates that were formed by templating PS beads against 2D arrays of cylindrical holes. The cylindrical holes used in all experiments are $\cong 2 \mu \mathrm{m}$. (a) Dimers with $1.0 \mu \mathrm{m}$ PS beads; (b) trimers with $0.9 \mu \mathrm{m}$ PS beads; (c) square tetramers with $0.8 \mu \mathrm{m}$ PS beads, and (d) pentagons with $0.7 \mu \mathrm{m}$ PS beads. "Reprinted with permission from [116]. Copyright (1996) American Chemical Society."
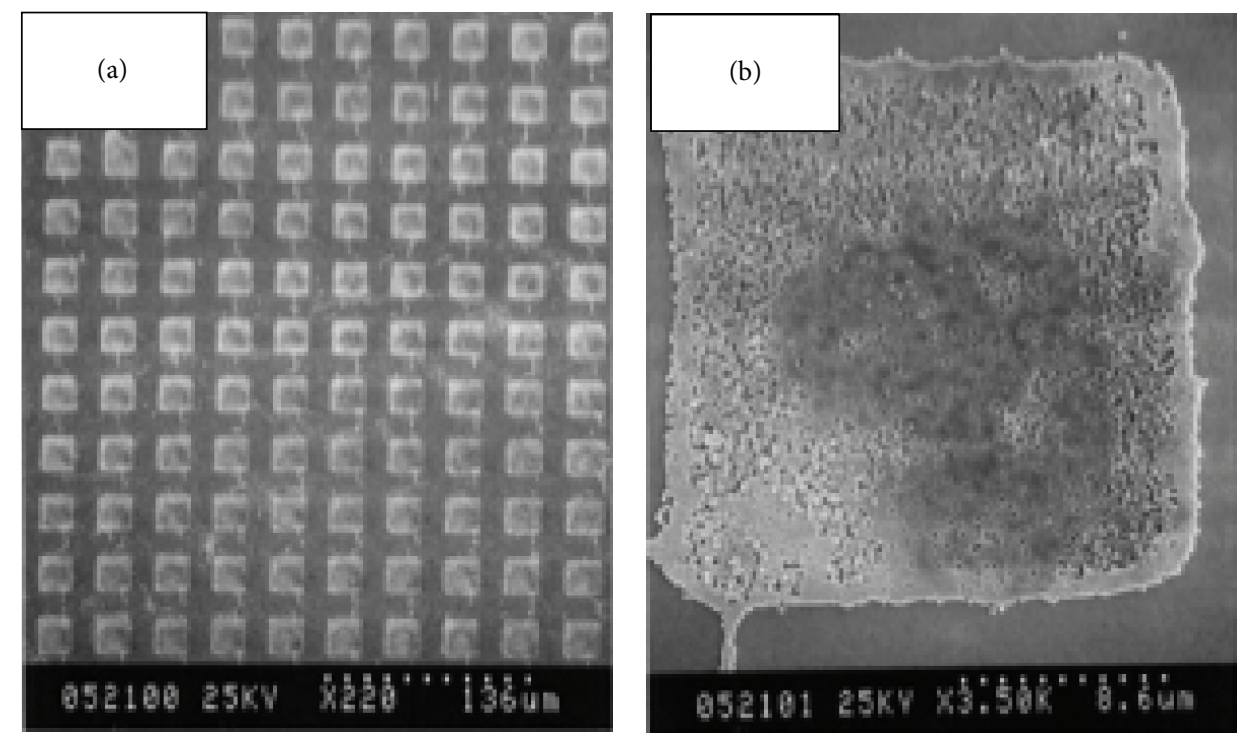

FIGURE 15: SEM micrographs of a chemical patterning experiment by use of a square elastomer stamp as structure for printing. (a) Overview of the sample, (b) zoom on one square. Dimensions of the pattern: squares $(24 \mu \mathrm{m})^{2}$. "Reproduced from [83] with permission of the PCCP Owner Societies." 

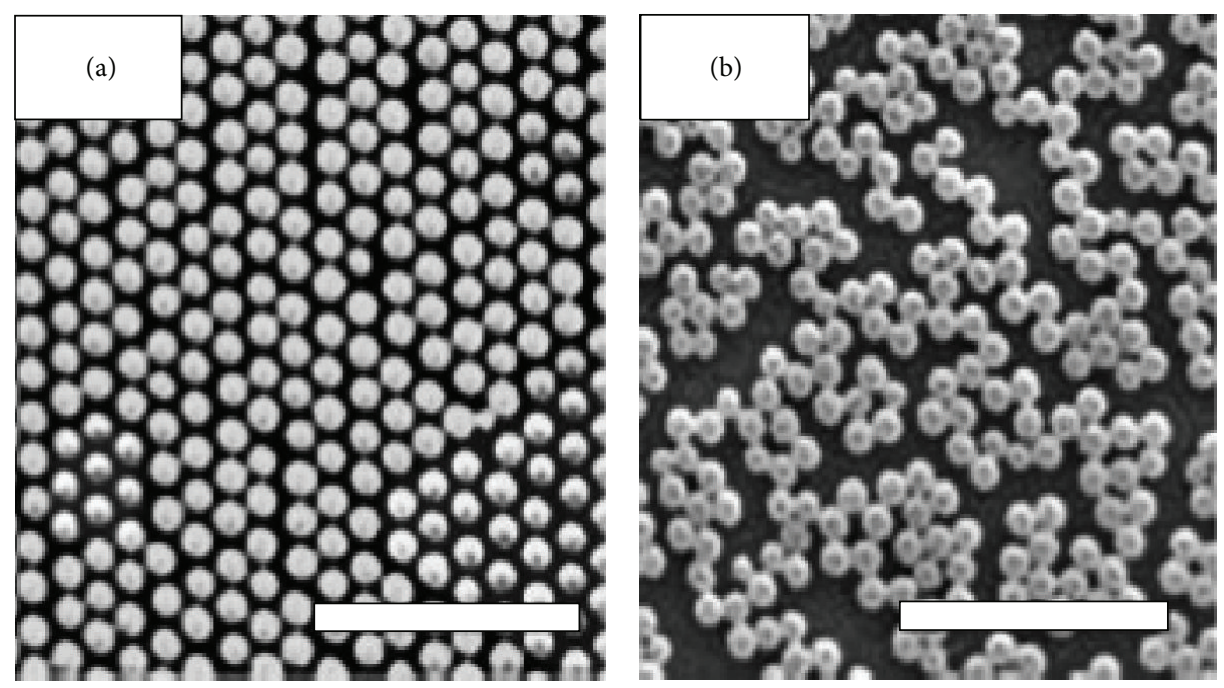

FIGURE 16: SEM micrographs of 2D colloidal crystals grown by electrophoretic deposition on (a) a patterned and on (b) an unpatterned ITO substrate. Scale bars are $5 \mu \mathrm{m}$. "Reprinted with permission from [81]. Copyright (2004) John Wiley and Sons."

a substrate. The deposited materials can be chosen without almost any limitation and the feature size of obtained structures can be controlled by adjusting the particle diameters, ranging from less than ten nanometers [85] to tens of micrometers [86].

Although the preparation of a colloidal mask is easy and cheap, the resulting pattern is limited to triangular structures, which can be a significant drawback, compared to conventional lithographic techniques. Indeed, in many applications, the material properties and target applications are highly dependent on the shape of the patterned species. To overcome this disadvantage and build new architectures, various strategies have been suggested and will also briefly discussed in this review.

3.1. Nanodots. Nanoparticle arrays can simply be generated by deposition on the substrate through the mask, which is usually positioned normal to the propagation direction of a material vapor. The particles obtained after removal of the mask have, in general, approximate triangular shape (Figure 17(a)) and are distributed in hexagonal lattices at the substrate surface.

Among all applications of those structures, localized surface plasmon resonance (LSPR) [87] is one active area of great interest. LSPR plays a major role in new developments of nanophotonics, such as surface enhanced Raman scattering $[88,89]$ (SERS) substrates or biosensors [90]. Haynes and Van Duyne [88] investigated the relationship between particles sizes, shape, interparticle spacing, and LSPR. They worked on hexagonally ordered triangular Ag dot arrays obtained with single-layered colloidal masks or Ag nanoparticle arrays obtained by double-layered colloidal masks (Figure 17). They demonstrated that the absorption band of the metallic nanostructures can be easily tuned from visible to near IR.

Another important area of the NSL is the design of ordered nanostructures for ultrahigh density magnetic recording [69, 91-93]. Compared with conventional techniques for fabricating magnetic ordered nanostructures, NSL is inexpensive and allows high-output fabrication. In general, reducing the feature dimensions to lower length scales has revealed novel magnetic properties [29]. For example, nanoscale magnetic materials often exhibit superparamagnetic behavior, which hinders the miniaturization race.

NSL allows a fine-tuning of the size of the manufactured nanostructures. Indeed, a number of posttreatment methods (e.g., heating (Figure 18) and etching) have been developed in order to modify the interstitial structure of the colloidal mask.

Haynes and coworkers [94] also pioneered angle-resolved colloidal lithography, which consists in the deposition of materials at the nonzero incident angle $(\theta)$ of the vapor beam with respect to the normal direction of the substrate. This technique not only reduced the dimension of the nanodots but also modified the shape from equilaterals to isosceles triangles (Figure 19).

Recently, Wang and coworkers broadened the scope of angle-resolved colloidal lithography by developing a stepwise strategy to generate heterogeneous binary particles (Figure 20).

3.2. Nanodisks-Nanorings. A modified colloidal lithography (hole-mask colloidal lithography [95]) enables the preparation of metal nanodisk arrays after several deposition/etching steps.

The essential new feature of hole-mask colloidal lithography compared to the original colloidal lithography is the presence of a sacrificial layer combined with a thin film mask with nanoholes ("hole mask"). A sacrificial polymer film (e.g., PMMA) is spin-coated onto a flat surface (e.g., glass slide and $\mathrm{Si}$ wafer). This is followed by deposition of a colloidal solution containing PS bead. A thin film, which is resistant to reactive oxygen plasma etching, is then deposited onto the surface (and not under the nanospheres). The PS beads 

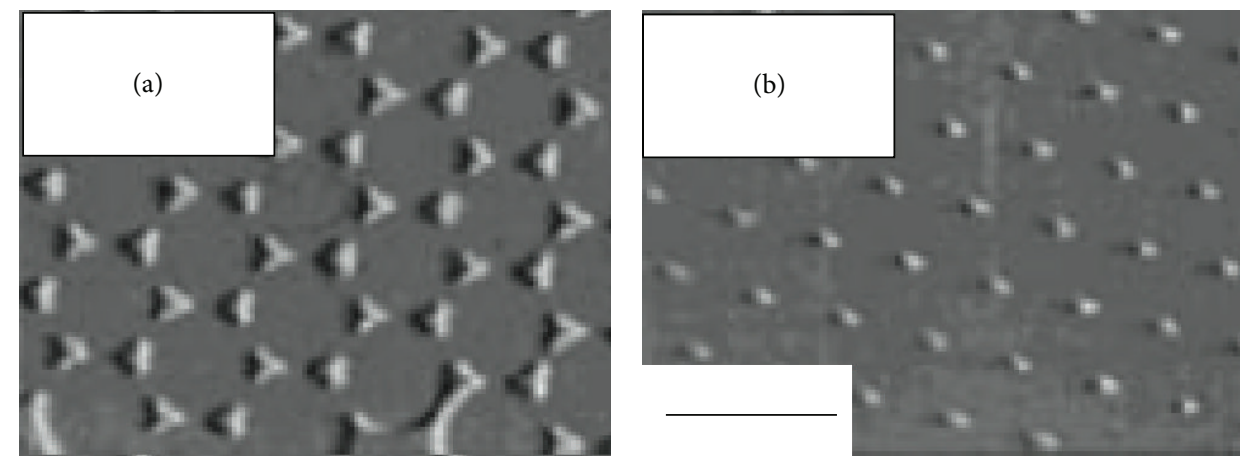

FIGURE 17: (a) SEM micrograph of Ag triangular nanodots made with a single-layered colloidal mask. (b) SEM micrograph of Ag nanodots made with a double-layered colloidal mask. The scale bar is $1 \mu \mathrm{m}$. "Reprinted with permission from [88]. Copyright (2001) American Chemical Society."

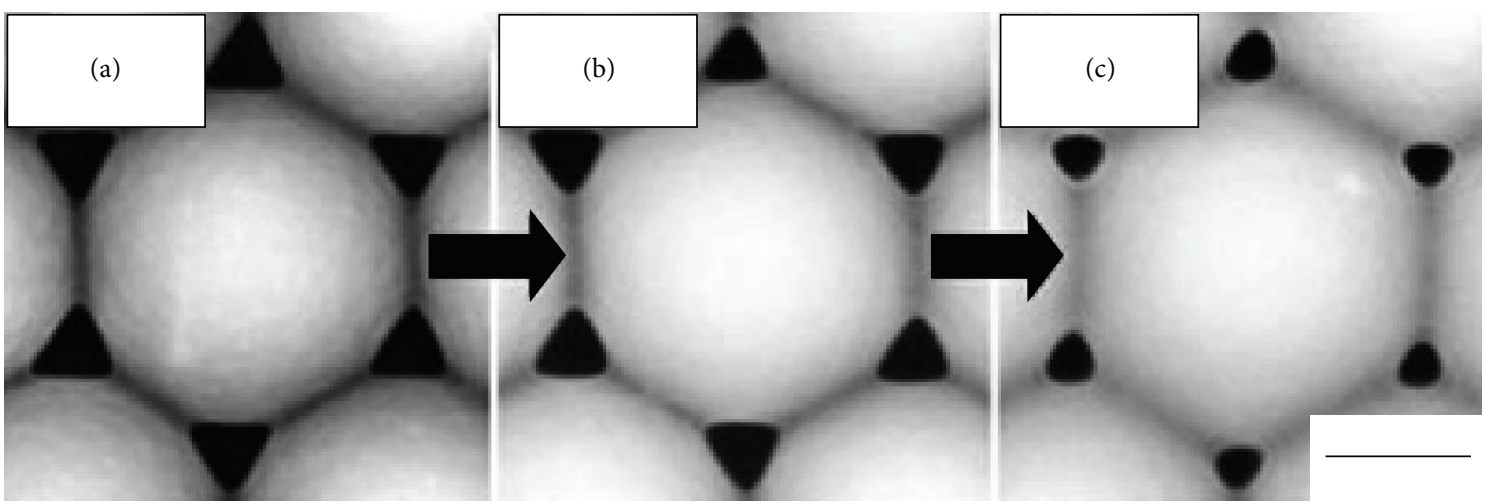

FIGURE 18: SEM micrographs of a PS mask annealed in water/EtOH/acetone mixture by (a) 1, (b) 2, and (c) 4 microwave pulses. The scale bar is $200 \mathrm{~nm}$. "Reprinted with permission from [117]. Copyright (2005) John Wiley and Sons."
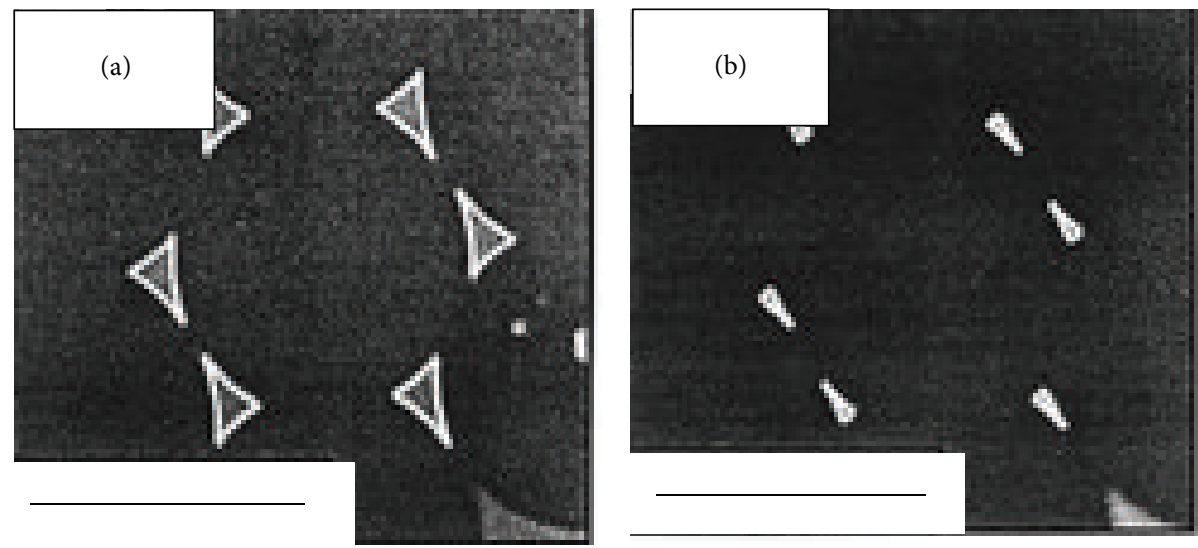

FIGURE 19: SEM micrographs of gold nanodots fabricated by angle-resolved colloidal lithography with (a) $\theta=20^{\circ}$ and (b) $\theta=40^{\circ}$. The scale bar is $1 \mu \mathrm{m}$. "Reprinted with permission from [94]. Copyright (2002) American Chemical Society."

are tape-stripped away and the film is then subjected to plasma treatment, leaving nanoholes in the plasma-resistant film ("hole mask") resting on the sacrificial PMMA layer. The hole mask can then be used either as a deposition- or etchmask or both.

Kasemo and his group fabricated Pt [96], Pd [96], Ag [96], and $\mathrm{Al}$ [97] nanodisks (Figure 21) and studied their plasmonic properties.
Choi et al. [98] studied nanopatterned Co/Pt nanodisks for magnetic memory devices.

Aizpurua et al. [99] investigated the optical properties of $\mathrm{Au}$ nanorings using another modified colloidal lithography, coupled with an Ar ion beam etching step. A red-shifted localized surface plasmon was observed compared to $\mathrm{Au}$ nanodisks of similar size. Those nanorings may also be used as ultrasensitive biosensors [100]. 

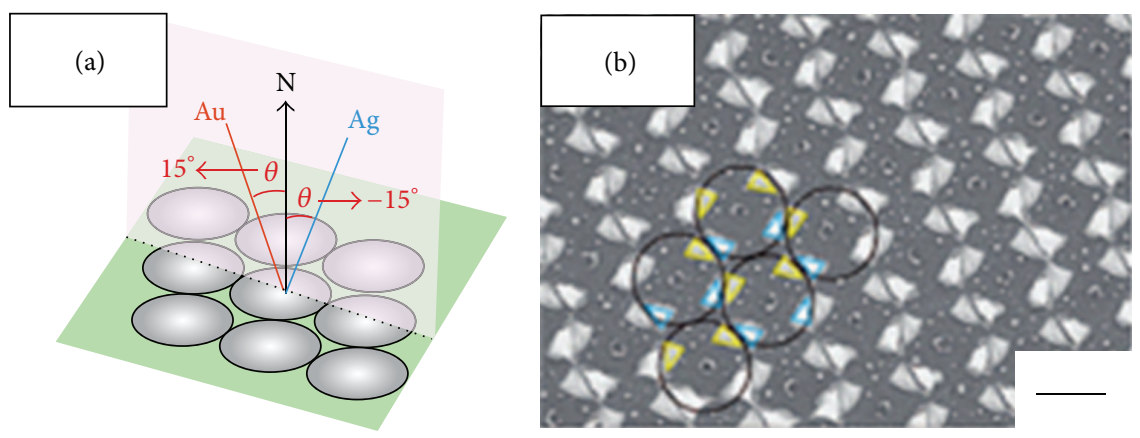

FIGURE 20: (a) Schematic illustration of depositing gold and silver onto a hexagonally close-packed spheres monolayer at the incidence angles $(\theta)$ of 15 and $-15^{\circ}$, respectively. The incident beam of gold, the incident beam of silver, and the normal direction of the colloidal template are highlighted by yellow, blue, and black arrows, respectively. (b) SEM image of the resulting heterogeneous binary array. The original locations of PS spheres, gold NPs, and silver NPs are highlighted by black circles, yellow triangles, and blue triangles, respectively. The scale bar is $2 \mu \mathrm{m}$. "Reprinted with permission from [118]. Copyright (2008) American Chemical Society."
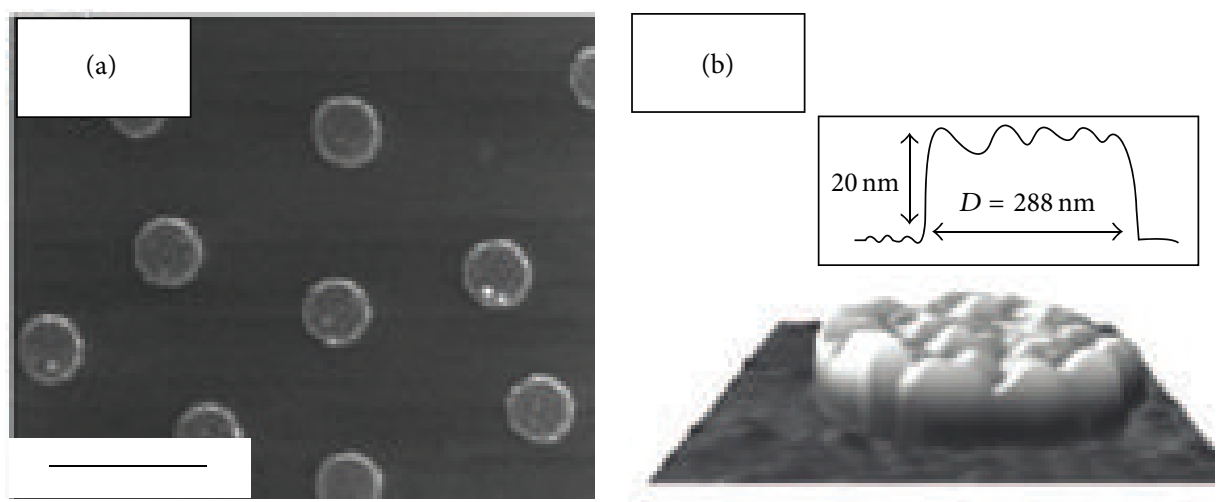

Figure 21: (a) SEM micrograph of Pt nanodisks. The scale bar is $500 \mathrm{~nm}$. "Reprinted with permission from [96]. Copyright (2006) American Chemical Society." (b) AFM 3D micrograph of a Al nanodisk. Cross-section profile reveals a rather rough surface. "Reprinted with permission from [97]. Copyright (2008) American Chemical Society."

A dewetting process also enables the synthesizing of nanorings of various materials $\left(\mathrm{TiO}_{2}[101]\right.$, CdSe Quantum Dots [102], and proteins [103]). First, a solution containing the desired material is filled into a colloidal template on a substrate. During the evaporation of solvent at the nanosphere/substrate interface, the solvent molecules around the contact area usually evaporate last, because they are confined by capillary forces. The materials dissolved or dispersed in the solvent move towards these areas and form ring-like structures under the nanospheres (Figure 22).

3.3. Nanoholes. The fabrication of nanoholes is based on the modification of colloidal masks by etching or heating treatment. It modulates the size of the interstitial pores between the particles, changing the arrangement from close packing to nonclose packing.

This technology allows considerable freedom to control both the feature dimensions and shape of nanoholes by changing the sizes of nanospheres. By filling the empty interspace between nanospheres with target materials, nanohole or nanopore arrays can be produced. Periodic metallic nanohole arrays (Figures 23 and 24), as other metallic nanostructures, have attracted great interest because of their important technological applications in nanophotonic devices.

Current challenge in this field lies in the fabrication of nonspherical nanohole arrays (Figure 24) due to the fact that the shape of the individual nanoholes offers an important handle to tune the plasmonic properties.

Recently, Yu et al. [104] developed a facile and new approach to prepare large-scale ordered porous nanoparticles (e.g., gold or $\mathrm{Fe}_{3} \mathrm{O}_{4}$ ) films, by filling the nanoparticles into interstitial voids of the sphere arrays by filtration technique. Then the ordered porous structured were formed after heating and removing the sphere template (Figure 25). The obtained structures can be used for membranes, catalysts, and surface enhanced Raman scattering probes.

3.4. Nanorods-Nanowires-Nanopillars-Nanotips. By catalytic deposition or growth, 1D structured arrays can be fabricated using 2D colloidal crystals as templates.

Metal nanoparticle arrays can be used as catalysts to grow 1D nanowire arrays. Indeed, Ni nanoparticle arrays have been used to prepare carbon nanotube arrays (Figure 26) by plasma-enhanced chemical vapor deposition (PECVD). 

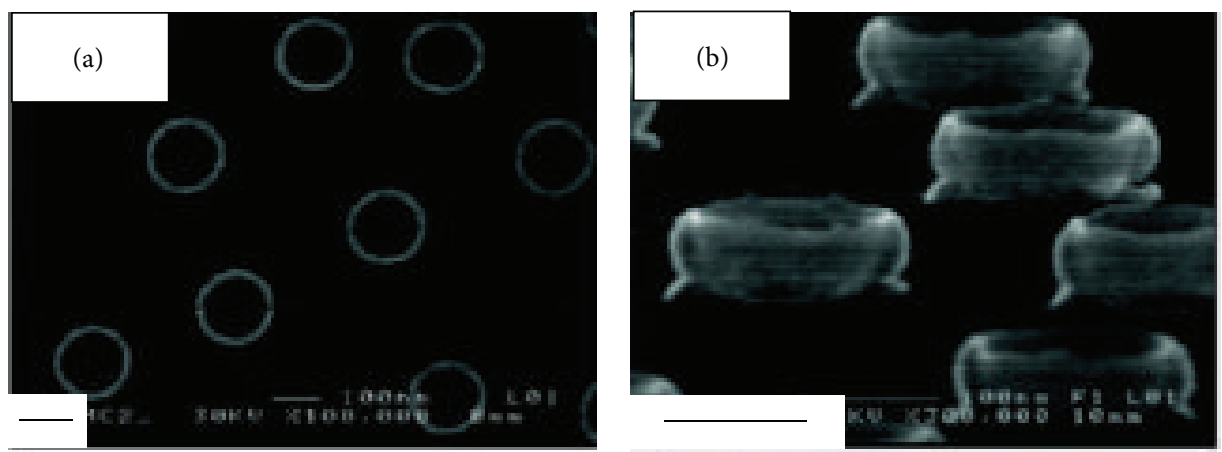

FIGURE 22: SEM micrographs of Au nanorings: (a) top view and (b) $80^{\circ}$ tilted view. Both scale bars are 100 nm. "Reprinted with permission from [100]. Copyright (2007) American Chemical Society."

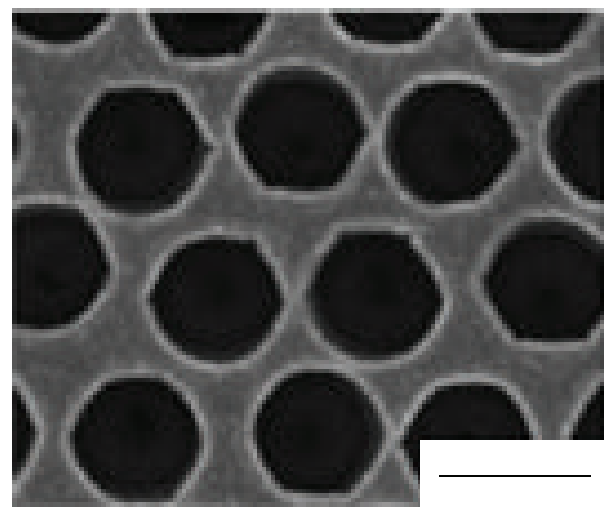

FIGURE 23: SEM micrograph of Ni porous ordered arrays. The scale bar is $1 \mu \mathrm{m}$. "Reprinted with permission from [119]. Copyright (2009) American Chemical Society."
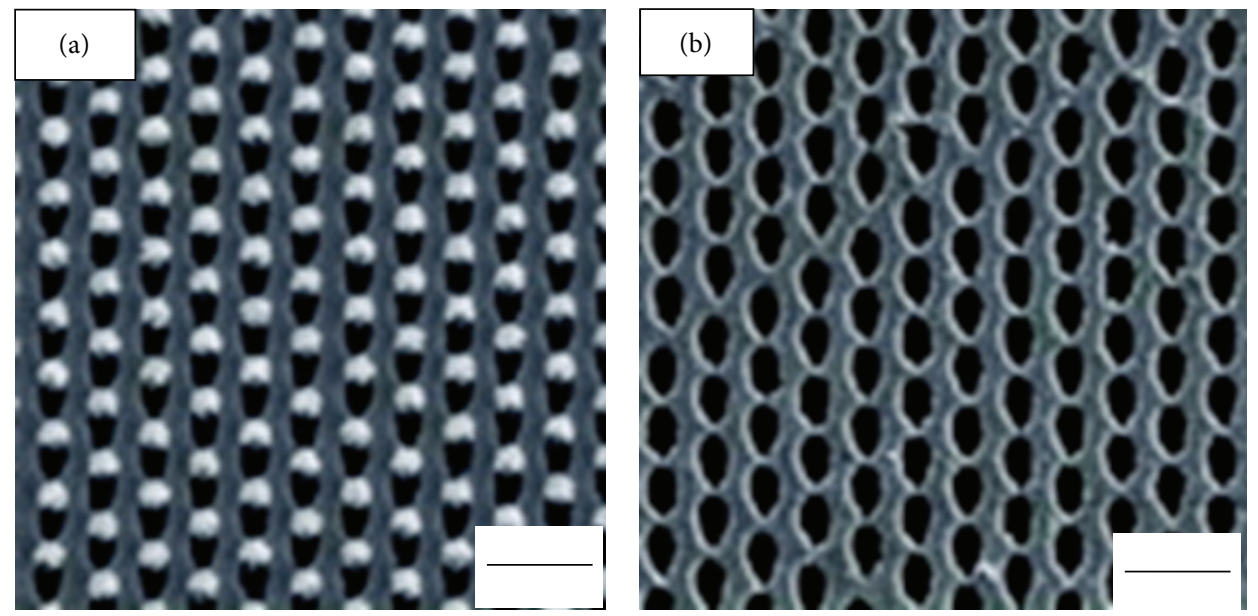

FIGURE 24: SEM micrographs of elliptical Ag porous ordered arrays before (a) and after (b) nanosphere removal. The scale bar is $1 \mu \mathrm{m}$. "Reprinted with permission from [120]. Copyright (2009) American Chemical Society."

Wang and coworkers [105] prepared aligned $\mathrm{ZnO}$ nanorods by vapor-liquid-solid growth guided by $\mathrm{Au}$ nanoparticles arrays.

$\mathrm{Li}$ and coworkers [106] reported a new approach for manufacturing $\mathrm{TiO}_{2}$ hcp nanocolumn arrays (Figure 27(a)) by pulsed laser deposition (PLD) in oxygen on the top of PS nanospheres. The periodicity can be tuned by using different nanosphere sizes and the distance between neighboring columns could be controlled by experiment parameters (e.g., pressure) during process (Figure 27).

By changing the corresponding target in the PLD process, this strategy can be used to synthesize similar nanocolumns 


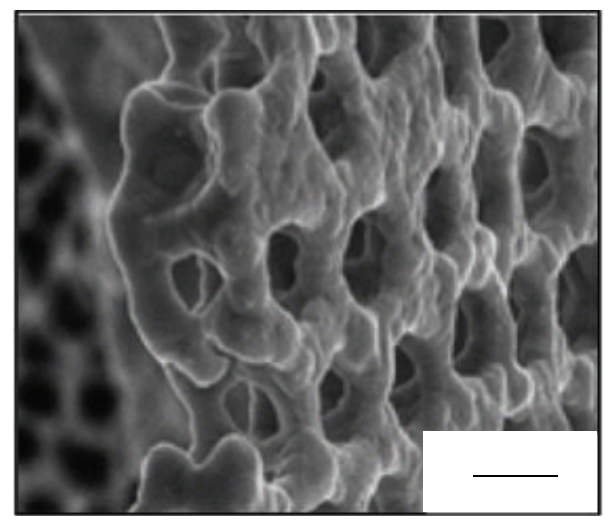

FIGURE 25: Cross-sectional SEM image (after removal of the $1.09 \mu \mathrm{m}$ polystyrene monolayer template) of a film prepared from $100 \mathrm{~nm}$ gold nanoparticles. The scale bar is $500 \mathrm{~nm}$. "Reprinted from [104] with permission of the Royal Society of Chemistry."

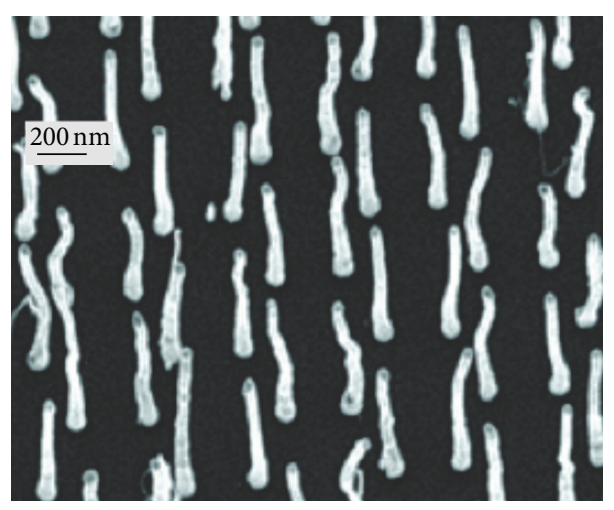

FIGURE 26: Tilted SEM micrograph of aligned carbon nanotubes (CNTs). "Reprinted with permission from [121]. Copyright (2003) American Chemical Society."
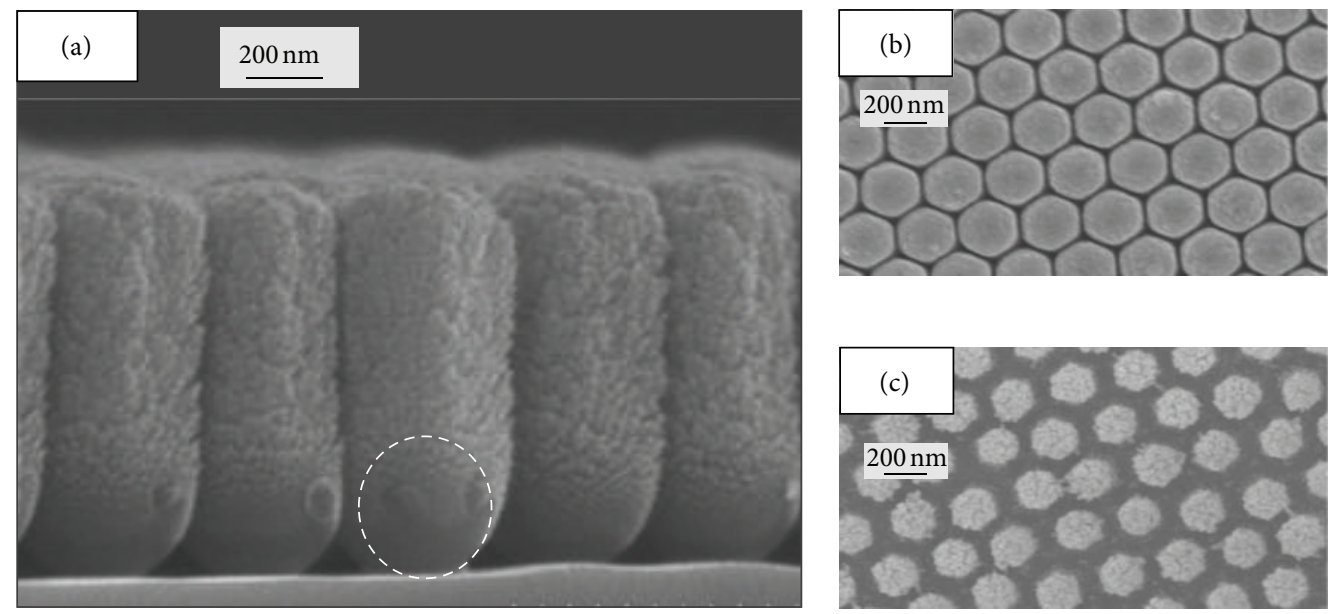

FIGURE 27: $\mathrm{TiO}_{2}$ nanocolumn array obtained by PLD using a PS colloidal monolayer as substrate. (a) HR-SEM micrograph observed from the side (the dotted circle highlights the PS sphere). "Adapted with permission from [122]. Copyright (2008) American Chemical Society." (b), (c) Top-view HR-SEM micrograph of $\mathrm{TiO}_{2}$ nanorods obtained under different background gas pressures ((b) $2.0 \mathrm{~Pa}$ for $200 \mathrm{~min}$ and (c) 26.7 Pa for $30 \mathrm{~min}$ ) and subsequent annealing. "Adapted with permission from [123]. Copyright (2005) John Wiley and Sons." 

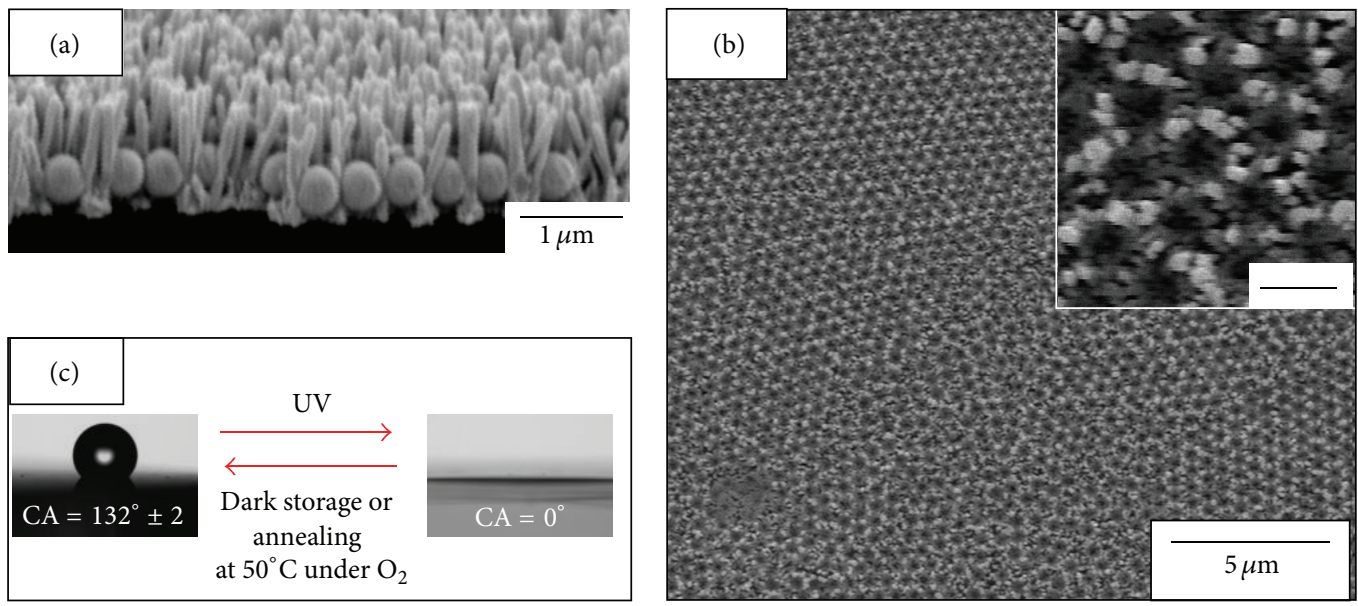

FIGURE 28: ZnO nanowire arrays grown via the "templated growth" route trough a sacrificial layer of polystyrene nanospheres, showing controllable and reversible wetting properties. (a) Cross-section SEM micrograph showing the as-grown ZnO nanowires through the polystyrene nanospheres. (b) SEM micrograph of the $\mathrm{ZnO}$ nanowires after removal of the polystyrene template, highlighting a hexagonal symmetry. The inset scale bar is $500 \mathrm{~nm}$. (c) Reversible hydrophobicity to superhydrophilicity by successive UV illumination and dark storage or annealing (CA: contact angle). "Adapted with permission from [109]. Copyright (2011) American Chemical Society."
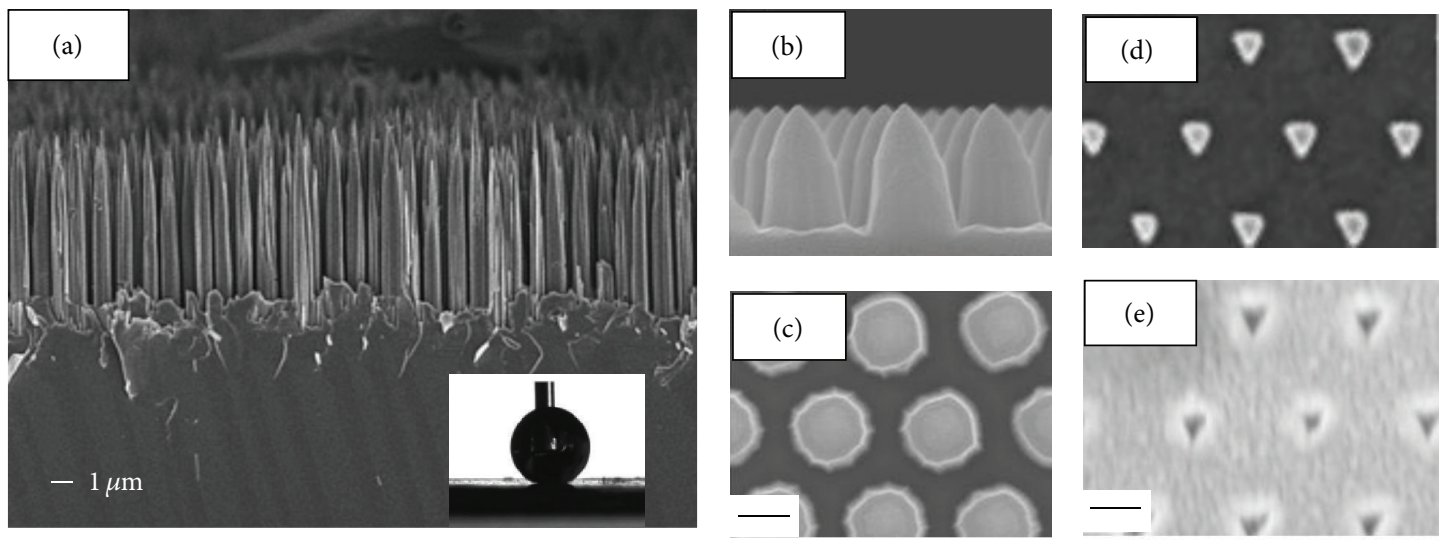

FIGURE 29: (a) SEM micrograph of a cross-section of the silicon tip arrays obtained by wet etching. The inset is the profile of water droplet on the sample. "Reproduced from [110] with permission of the Royal Society of Chemistry." (b), (c) Respectively side and top SEM micrographs of RIE-etched silicon sample for antireflective applications. The scale bar is $200 \mathrm{~nm}$. "Reprinted with permission from [113]." (d) SEM micrograph of silicon nanoimprint lithography stamp obtained by RIE etching. (e) SEM micrograph of corresponding imprinted pattern on PMMA. The scale bar is $200 \mathrm{~nm}$. "Reprinted with permission from [124]. Copyright (2003) John Wiley and Sons."

in different materials such as $\mathrm{Co}_{3} \mathrm{O}_{4},[107] \mathrm{ZnO},[108] \mathrm{Fe}_{2} \mathrm{O}_{3}$, [108], or $\mathrm{CuO}$ [108]. These nanostructures, in addition to intrinsic properties of materials they are made of, may display interesting wettability properties [107].

Our group also investigated the use of nanosphere lithography process to produce density-controlled patterned arrays of well-aligned $\mathrm{ZnO}$ nanowires by hydrothermal growth. Reversible superhydrophilicity to hydrophobicity was observed and controlled by successive UV illumination and $\mathrm{O}_{2}$ annealing (Figure 28) [109].

Two-dimensional colloidal crystals can also be used as protective masks to prepare nanotip/nanopillar arrays by etching procedures such as dry etching by reactive ion etching (RIE) or metal catalytic wet etching [110, 111].
RIE steps of certain materials are realized by corresponding ions, for example, polymer and carbon by oxygen ions, but silicon and silica by fluorine ions [112].

Nanotips and nanopillars are of great importance because of their unique potential in a wide variety of applications (Figure 29), such as antireflection coatings [110, 113], highly hydrophobic surfaces [110], and mold for nanoimprint lithography [114].

\section{Conclusions}

The complexity of the preparation process combined with high initial equipment costs made the conventional lithographic techniques unfavorable for many researchers. Moreover, most of the nonconventional lithographic techniques 
developed to date require the assistance of conventional lithographic techniques, such as photolithography, to design and manufacture the masks or masters.

In this context, nanosphere lithography emerged as a hybrid (between top-down and bottom-up approaches) and high-output nanostructure process:

(i) to produce nano objects, exhibiting similar features in terms of size, shape, and interface in order to exhibit similar (e.g., chemical, magnetic,etc.) properties;

(ii) to manufacture long range ordered arrays of nanostructures: all nanostructures are equidistant and have the same environment;

(iii) to control the density of these nanostructures in order to isolate or otherwise increase their number on a given surface;

(iv) to offer a certain flexibility in the choice of materials, both for the substrate and the nanostructures;

(v) to fulfill the above conditions in a short time and at reasonable price.

Throughout this review, we compared the various deposition techniques that can be used to manufacture the colloidal crystal masks, with respect to their advantages and drawbacks. Nanosphere lithography was here clearly evidenced as a powerful process to manufacture various arrays of periodic structures with a wide potential of interest for the field of nano- and microfabrication, as highlighted by the unexhaustive list of architectures/applications manufactured by NSL.

\section{References}

[1] R. P. Feynman, “There's plenty of room at the bottom," Engineering Science, vol. 23, no. 5, pp. 269-283, 1960.

[2] P. Xavier, "Les techniques de micro et nanofabrication," 2006, http://www.lirmm.fr/ w3mic/mns/presentation_Lille2006/xavier.pdf.

[3] H. Kurz, "Fabrication of micro- and nanostructures," 2006.

[4] C. Thibault, Impression de Biomolécules par Lithographie Douche, AppLications pour les Biopuces, de L'échelle Micrométrique á Nanométrique, Univeristé de TOULOUSE, 2007.

[5] "Nanoscience and nanotechnologies," 2004, http://www.nanotec.org.uk/report/chapter2.pdf.

[6] G. Agnus, T. Arnal, A. Fleurence et al., "Redécouvrir la matière-ordonner au nano," Plein Sud, http://www.pleinsud .u-psud.fr/specialR/04.pdf.

[7] A. Martinez-Gil, Nanostructuration de Surfaces Silicium pour Guider la Croissance Auto-Organisée de Nanostructures Métalliques, Université Paris-Sud XI, Orsay, France, 2005.

[8] W. Kandulski, Shadow nanosphere lithograpy [Ph.D. thesis], University of Bonn, 2007.

[9] R. W. Whatmore, "Nanotechnology: big prospects for small engineering," Ingenia, pp. 28-34, 2001.

[10] S. Okazaki, "Resolution limits of optical lithography," Journal of Vacuum Science \& Technology B, vol. 9, no. 6, pp. 2829-2833, 1991.
[11] Y. Xia and G. M. Whitesides, "Soft lithography," Angewandte Chemie, vol. 37, no. 5, pp. 550-575, 1998.

[12] "Soft lithography: reviews," http://www.chem.hanyang.ac .kr:8001/hanyang/professor6/upload/Lhito.pdf.

[13] B. D. Gates, Q. Xu, M. Stewart, D. Ryan, C. G. Willson, and G. M. Whitesides, "New approaches to nanofabrication: molding, printing, and other techniques," Chemical Reviews, vol. 105, no. 4, pp. 1171-1196, 2005.

[14] P. W. K. Rothemund, "Folding DNA to create nanoscale shapes and patterns," Nature, vol. 440, no. 7082, pp. 297-302, 2006.

[15] C. Martin, Développement, par une Approche Mixte Top-Down/Bottom-Up, de Dispositifs Planaires pour la Nanoélectronique, INSA Toulouse, 2005.

[16] R. Garcia, R. V. Martinez, and J. Martinez, "Nano-chemistry and scanning probe nanolithographies," Chemical Society Reviews, vol. 35, no. 1, pp. 29-38, 2006.

[17] D. M. Eigler and E. K. Schweizer, "Positioning single atoms with a scanning tunnelling microscope," Nature, vol. 344, no. 6266, pp. 524-526, 1990.

[18] M. Zhang, D. Bullen, S.-W. Chung et al., "A MEMS nanoplotter with high-density parallel dip-pen nanolithography probe arrays," Nanotechnology, vol. 13, no. 2, pp. 212-217, 2002.

[19] M. Geissler and Y. Xia, "Patterning: principles and some new developments," Advanced Materials, vol. 16, no. 15, pp. 1249$1269,2004$.

[20] B. D. Gates, Q. Xu, J. C. Love, D. B. Wolfe, and G. M. Whitesides, "Unconventional nanofabrication," Annual Review of Materials Research, vol. 34, pp. 339-372, 2004.

[21] T. Itani, W. Wakamiya, J. Cashmore, and M. Gower, "157-nm lithography with high numerical aperture lens for sub-70 nm node," Microelectronic Engineering, vol. 67-68, pp. 39-46, 2003.

[22] IBM. 20-02-2006, http://www-03.ibm.com/press/us/en/ pressrelease/19260.wss\#release.

[23] D. Bratton, D. Yang, J. Dai, and C. K. Ober, "Recent progress in high resolution lithography," Polymers for Advanced Technologies, vol. 17, no. 2, pp. 94-103, 2006.

[24] J.-M. Lehn, "Perspectives in supramolecular chemistry-from molecular recognition towards molecular information processing and self-organization," Angewandte Chemie, vol. 29, no. 11, pp. 1304-1319, 1990.

[25] J. C. Love, L. A. Estroff, J. K. Kriebel, R. G. Nuzzo, and G. M. Whitesides, "Self-assembled monolayers of thiolates on metals as a form of nanotechnology," Chemical Reviews, vol. 105, no. 4, pp. 1103-1169, 2005.

[26] J. C. Meiners, H. Elbs, A. Ritzi, J. Mlynek, and G. Krausch, "Chemically functionalized surfaces from ultrathin blockcopolymer films," Journal of Applied Physics, vol. 80, no. 4, pp. 2224-2227, 1996.

[27] G. Krausch and R. Magerle, "Nanostructured thin films via selfassembly of block copolymers," Advanced Materials, vol. 14, no. 21, pp. 1579-1583, 2002.

[28] M. Lazzari and M. Arturo López-Quintela, "Block copolymers as a tool for nanomaterial fabrication," Advanced Materials, vol. 15, no. 19, pp. 1583-1594, 2003.

[29] J. I. Martín, J. Nogués, K. Liu, J. L. Vicent, and I. K. Schuller, "Ordered magnetic nanostructures: fabrication and properties," Journal of Magnetism and Magnetic Materials, vol. 256, no. 1-3, pp. 449-501, 2003.

[30] J. Dutta and H. Hofmann, "Self-organization of colloidal nanoparticles," Encyclopedia of Nanoscience and Nanotechnology, vol. 9, pp. 617-640, 2004. 
[31] M. P. Pileni, Y. Lalatonne, D. Ingert, I. Lisiecki, and A. Courty, "Self assemblies of nanocrystals: preparation, collective properties and uses," Faraday Discussions, vol. 125, pp. 251-264, 2004.

[32] T.-W. Huang, K.-L. Yu, Y.-F. Liao, and C.-H. Lee, "Enhance the structural stability of the FePt nanoparticle monolayer by adding gold overlayer," Colloids and Surfaces A, vol. 284-285, pp. 603-606, 2006.

[33] P. Pieranski, "Two-dimensional interfacial colloidal crystals," Physical Review Letters, vol. 45, no. 7, pp. 569-572, 1980.

[34] G. Zhang and D. Wang, "Colloidal lithography-the art of nanochemical patterning," Chemistry, vol. 4, no. 2, pp. 236-245, 2009.

[35] S.-M. Yang, S. G. Jang, D.-G. Choi, S. Kim, and H. K. Yu, "Nanomachining by colloidal lithography," Small, vol. 2, no. 4, pp. 458-475, 2006.

[36] U. C. Fischer and H. P. Zingsheim, "Submicroscopic pattern replication with visible light," Journal of Vacuum Science \& Technology, vol. 19, no. 4, pp. 881-885, 1981.

[37] H. W. Deckman and J. H. Dunsmuir, "Natural lithography," Applied Physics Letters, vol. 41, no. 4, pp. 377-379, 1982.

[38] H. W. Deckman and J. H. Dunsmuir, "Applications of surface textures produced with natural lithography," Journal of Vacuum Science and Technology B, vol. 1, no. 4, pp. 1109-1112, 1983.

[39] J. C. Hulteen and R. P. Van Duyne, "Nanosphere lithography: a materials general fabrication process for periodic particle array surfaces," Journal of Vacuum Science and Technology A, vol. 13, no. 3, pp. 1553-1558, 1995.

[40] T. R. Jensen, G. C. Schatz, and R. P. Van Duyne, "Nanosphere lithography: surface plasmon resonance spectrum of a periodic array of silver nanoparticles by ultraviolet-visible extinction spectroscopy and electrodynamic modeling," Journal of Physical Chemistry B, vol. 103, no. 13, pp. 2394-2401, 1999.

[41] X. Zhang, C. R. Yonzon, and R. P. Van Duyne, "Nanosphere lithography fabricated plasmonic materials and their applications," Journal of Materials Research, vol. 21, no. 5, pp. 1083-1092, 2006.

[42] W. B. Russel, D. A. Saville, and W. R. Schowalter, Colloidal Dispersions, Cambridge University Press, Cambridge, UK, 1989.

[43] L. Li, T. Zhai, H. Zeng, X. Fang, Y. Bando, and D. Golberg, "Polystyrene sphere-assisted one-dimensional nanostructure arrays: synthesis and applications," Journal of Materials Chemistry, vol. 21, no. 1, pp. 40-56, 2011.

[44] Y. Li, N. Koshizaki, and W. Cai, "Periodic one-dimensional nanostructured arrays based on colloidal templates, applications, and devices," Coordination Chemistry Reviews, vol. 255, no. 3-4, pp. 357-373, 2011.

[45] Y. Li, W. Cai, and G. Duan, "Ordered micro/nanostructured arrays based on the monolayer colloidal crystals," Chemistry of Materials, vol. 20, no. 3, pp. 615-624, 2008.

[46] J. Dewalque, R. Cloots, O. Dubreuil, N. Krins, B. Vertruyen, and C. Henrist, "Microstructural evolution of a $\mathrm{TiO}_{2}$ mesoporous single layer film under calcination: effect of stabilization and repeated thermal treatments on the film crystallization and surface area," Thin Solid Films, vol. 520, no. 16, pp. 5272-5276, 2012.

[47] X. Ye and L. Qi, “Two-dimensionally patterned nanostructures based on monolayer colloidal crystals: controllable fabrication, assembly, and applications," Nano Today, vol. 6, no. 6, pp. 608631, 2011.

[48] N. D. Denkov, O. D. Velev, P. A. Kralchevsky, I. B. Ivanov, H. Yoshimura, and K. Nagayama, "Mechanism of formation of two-dimensional crystals from latex particles on substrates," Langmuir, vol. 8, no. 12, pp. 3183-3190, 1992.

[49] P. A. Kralchevsky and N. D. Denkov, "Capillary forces and structuring in layers of colloid particles," Current Opinion in Colloid and Interface Science, vol. 6, no. 4, pp. 383-401, 2001.

[50] R. Micheletto, H. Fukuda, and M. Ohtsu, "A simple method for the production of a two-dimensional, ordered array of small latex particles," Langmuir, vol. 11, no. 9, pp. 3333-3336, 1995.

[51] Z. Zhong, H. Zhang, X. Tang, Y. Jing, L. Zhang, and S. Liu, "Fabrication of submicron magnetic oxide antidot arrays by combining nanosphere lithography with sputtering technology," Physica E, vol. 40, no. 3, pp. 516-519, 2008.

[52] T. Ning, Y. Zhou, H. Shen et al., "Nonlinear optical properties of $\mathrm{Au} / \mathrm{ZnO}$ nanoparticle arrays," Applied Surface Science, vol. 254, no. 7, pp. 1900-1903, 2008.

[53] A. S. Dimitrov and K. Nagayama, "Continuous convective assembling of fine particles into two-dimensional arrays on solid surfaces," Langmuir, vol. 12, no. 5, pp. 1303-1311, 1996.

[54] R. C. Rossi, M. X. Tan, and N. S. Lewis, "Size-dependent electrical behavior of spatially inhomogeneous barrier height regions on silicon," Applied Physics Letters, vol. 77, no. 17, pp. 2698-2700, 2000.

[55] D. Nagao, R. Kameyama, Y. Kobayashi, and M. Konno, "Multiformity of particle arrays assembled with a simple dip-coating," Colloids and Surfaces A, vol. 311, no. 1-3, pp. 26-31, 2007.

[56] Y. B. Zheng, S. J. Chua, C. H. A. Huan, and Z. L. Miao, "Selective growth of GaAs quantum dots on the triangle nanocavities bounded by $\mathrm{SiO}_{2}$ mask on $\mathrm{Si}$ substrate by MBE," Journal of Crystal Growth, vol. 268, no. 3-4, pp. 369-374, 2004.

[57] C.-W. Kuo, J.-Y. Shiu, and P. Chen, "Size- and shape-controlled fabrication of large-area periodic nanopillar arrays," Chemistry of Materials, vol. 15, no. 15, pp. 2917-2920, 2003.

[58] E. C. Brown, S. K. Wilke, D. A. Boyd, D. G. Goodwin, and S. M. Haile, "Polymer sphere lithography for solid oxide fuel cells: a route to functional, well-defined electrode structures," Journal of Materials Chemistry, vol. 20, no. 11, pp. 2190-2196, 2010.

[59] P. Colson, R. Cloots, and C. Henrist, "Experimental design applied to spin coating of $2 \mathrm{~d}$ colloidal crystal masks: a relevant method?” Langmuir, vol. 27, no. 21, pp. 12800-12806, 2011.

[60] T. Rehg and B. Higgins, "Spin coating of colloidal suspensions," AIChE Journal, vol. 38, no. 4, pp. 489-501, 1992.

[61] P. Jiang and M. J. McFarland, "Large-scale fabrication of wafersize colloidal crystals, macroporous polymers and nanocomposites by spin-coating," Journal of the American Chemical Society, vol. 126, no. 42, pp. 13778-13786, 2004.

[62] N. D. Denkov, O. D. Velev, P. A. Kralchevsky, I. B. Ivanov, H. Yoshimura, and K. Nagayama, "Mechanism of formation of two-dimensional crystals from latex particles on substrates," Langmuir, vol. 8, no. 12, pp. 3183-3190, 1992.

[63] S. Middleman, "The effect of induced air-flow on the spin coating of viscous liquids," Journal of Applied Physics, vol. 62, no. 6, pp. 2530-2532, 1987.

[64] C. D. Dushkin, G. S. Lazarov, S. N. Kotsev, H. Yoshimura, and K. Nagayama, "Effect of growth conditions on the structure of twodimensional latex crystals: experiment," Colloid and Polymer Science, vol. 277, no. 10, pp. 914-930, 1999.

[65] J. Chen, P. Dong, D. Di et al., "Controllable fabrication of 2D colloidal-crystal films with polystyrene nanospheres of various diameters by spin-coating," Applied Surface Science, vol. 270, pp. 6-15, 2013. 
[66] J. Rybczynski, U. Ebels, and M. Giersig, "Large-scale, 2D arrays of magnetic nanoparticles," Colloids and Surfaces A, vol. 219, no. 1-3, pp. 1-6, 2003.

[67] W.-D. Ruan, Z.-C. LÜ, N. Ji, C.-X. Wang, B. Zhao, and J.-H. Zhang, "Facile fabrication of large area polystyrene colloidal crystal monolayer via surfactant-free langmuir-blodgett technique," Chemical Research in Chinese Universities, vol. 23, no. 6, pp. 712-714, 2007.

[68] L. Zhang and Y. Xiong, "Rapid self-assembly of submicrospheres at liquid surface by controlling evaporation and its mechanism," Journal of Colloid and Interface Science, vol. 306, no. 2, pp. 428-432, 2007.

[69] S. M. Weekes, F. Y. Ogrin, W. A. Murray, and P. S. Keatley, "Macroscopic arrays of magnetic nanostructures from selfassembled nanosphere templates," Langmuir, vol. 23, no. 3, pp. 1057-1060, 2007.

[70] P. I. Stavroulakis, N. Christou, and D. Bagnall, "Improved deposition of large scale ordered nanosphere monolayers via liquid surface self-assembly," Materials Science and Engineering $B$, vol. 165, no. 3, pp. 186-189, 2009.

[71] L. M. Goldenberg, J. Wagner, J. Stumpe, B.-R. Paulke, and E. Görnitz, "Simple method for the preparation of colloidal particle monolayers at the water/alkane interface," Langmuir, vol. 18, no. 14, pp. 5627-5629, 2002.

[72] M. Giersig and P. Mulvaney, "Preparation of ordered colloid monolayers by electrophoretic deposition," Langmuir, vol. 9, no. 12, pp. 3408-3413, 1993.

[73] A. L. Rogach, N. A. Kotov, D. S. Koktysh, J. W. Ostrander, and G. A. Ragoisha, "Electrophoretic deposition of latex-based 3D colloidal photonic crystals: a technique for rapid production of high-quality opals," Chemistry of Materials, vol. 12, no. 9, pp. 2721-2726, 2000.

[74] M. Trau, D. A. Seville, and I. A. Aksay, "Field-induced layering of colloidal crystals," Science, vol. 272, no. 5262, pp. 706-709, 1996.

[75] Y. Solomentsev, M. Böhmer, and J. L. Anderson, "Particle clustering and pattern formation during electrophoretic deposition: a hydrodynamic model," Langmuir, vol. 13, no. 23, pp. 60586061, 1997.

[76] R. C. Hayward, D. A. Saville, and I. A. Aksay, "Electrophoretic assembly of colloidal crystals with optically tunable micropatterns," Nature, vol. 404, no. 6773, pp. 56-59, 2000.

[77] T. Gong and D. W. M. Marr, "Electrically switchable colloidal ordering in confined geometries," Langmuir, vol. 17, no. 8, pp. 2301-2304, 2001.

[78] S. O. Lumsdon, E. W. Kaler, and O. D. Velev, "Two-dimensional crystallization of microspheres by a coplanar AC electric field," Langmuir, vol. 20, no. 6, pp. 2108-2116, 2004.

[79] M. Bale, A. J. Turner, and R. E. Palmer, "Fabrication of ordered arrays of silicon nanopillars at selected sites," Journal of Physics $D$, vol. 35, no. 5, pp. L11-L14, 2002.

[80] Y. Xia, Y. Yin, Y. Lu, and J. McLellan, “Template-assisted selfassembly of spherical colloids into complex and controllable structures," Advanced Functional Materials, vol. 13, no. 12, pp. 907-918, 2003.

[81] M. Allard, E. H. Sargent, P. C. Lewis, and E. Kumacheva, "Colloidal crystals grown on patterned surfaces," Advanced Materials, vol. 16, no. 15, pp. 1360-1364, 2004.

[82] F. Fan and K. J. Stebe, "Assembly of colloidal particles by evaporation on surfaces with patterned hydrophobicity," Langmuir, vol. 20, no. 8, pp. 3062-3067, 2004.
[83] M. Himmelhaus and H. Takei, "Self-assembly of polystyrene nano particles into patterns of random-close-packed monolayers via chemically induced adsorption," Physical Chemistry Chemical Physics, vol. 4, no. 3, pp. 496-506, 2002.

[84] Q. Zhang, S. Ghosh, S. Samitsu, X. Peng, and I. Ichinose, "Ultrathin freestanding nanoporous membranes prepared from polystyrene nanoparticles," Journal of Materials Chemistry, vol. 21, no. 6, pp. 1684-1688, 2011.

[85] L. Y. Wu, B. M. Ross, and L. P. Lee, "Optical properties of the crescent-shaped nanohole antenna," Nano Letters, vol. 9, no. 5, pp. 1956-1961, 2009.

[86] H. Takei and N. Shimizu, "Gradient sensitive microscopic probes prepared by gold evaporation and chemisorption on latex spheres," Langmuir, vol. 13, no. 7, pp. 1865-1868, 1997.

[87] E. Hutter and J. H. Fendler, "Exploitation of localized surface plasmon resonance," Advanced Materials, vol. 16, no. 19, pp. 1685-1706, 2004.

[88] C. L. Haynes and R. P. Van Duyne, "Nanosphere lithography: a versatile nanofabrication tool for studies of size-dependent nanoparticle optics," Journal of Physical Chemistry B, vol. 105, no. 24, pp. 5599-5611, 2001.

[89] Y. Fang, N.-H. Seong, and D. D. Dlott, "Measurement of the distribution of site enhancements in surface-enhanced raman scattering," Science, vol. 321, no. 5887, pp. 388-392, 2008.

[90] A. J. Haes and R. P. Van Duyne, "A unified view of propagating and localized surface plasmon resonance biosensors," Analytical and Bioanalytical Chemistry, vol. 379, no. 7-8, pp. 920-930, 2004.

[91] S. M. Weekes, F. Y. Ogrin, and W. A. Murray, "Fabrication of large-area ferromagnetic arrays using etched nanosphere lithography," Langmuir, vol. 20, no. 25, pp. 11208-11212, 2004.

[92] H. Zhong, G. Tarrach, P. Wu, A. Drechsler, D. Wei, and J. Yuan, "High resolution magnetic force microscopy of patterned L10FePt dot arrays by nanosphere lithography," Nanotechnology, vol. 19, no. 9, Article ID 095703, 2008.

[93] J. Rybczynski, M. Hilgendorff, and M. Giersig, "Nanosphere lithography-fabrication of various periodic magnetic particle arrays using versatile nanosphere masks," in Low-Dimensional Systems: Theory, Preparation, and Some Applications, pp. 163172, 2003.

[94] C. L. Haynes, A. D. McFarland, M. T. Smith, J. C. Hulteen, and R. P. Van Duyne, "Angle-resolved nanosphere lithography: manipulation of nanoparticle size, shape, and interparticle spacing," Journal of Physical Chemistry B, vol. 106, no. 8, pp. 1898-1902, 2002.

[95] H. Fredriksson, Y. Alaverdyan, A. Dmitriev et al., "Hole-mask colloidal lithography," Advanced Materials, vol. 19, no. 23, pp. 4297-4302, 2007.

[96] C. Langhammer, Z. Yuan, I. Zorić, and B. Kasemo, "Plasmonic properties of supported Pt and Pd nanostructures," Nano Letters, vol. 6, no. 4, pp. 833-838, 2006.

[97] C. Langhammer, M. Schwind, B. Kasemo, and I. Zorić, "Localized surface plasmon resonances in aluminum nanodisks," Nano Letters, vol. 8, no. 5, pp. 1461-1471, 2008.

[98] D.-G. Choi, S. Kim, S.-G. Jang, S.-M. Yang, J.-R. Jeong, and S.-C. Shin, "Nanopatterned magnetic metal via colloidal lithography with reactive ion etching," Chemistry of Materials, vol. 16, no. 22, pp. 4208-4211, 2004.

[99] J. Aizpurua, P. Hanarp, D. S. Sutherland, M. Käll, G. W. Bryant, and F. J. García de Abajo, "Optical properties of gold nanorings," Physical Review Letters, vol. 90, no. 5, pp. 057401/1-057401/4, 2003. 
[100] E. M. Larsson, J. Alegret, M. Kǎll, and D. S. Sutherland, "Sensing characteristics of NIR localized surface plasmon resonances in gold nanorings for application as ultrasensitive biosensors," Nano Letters, vol. 7, no. 5, pp. 1256-1263, 2007.

[101] F. Sun, J. C. Yu, and X. Wang, "Construction of size-controllable hierarchical nanoporous $\mathrm{TiO}_{2}$ ring arrays and their modifications," Chemistry of Materials, vol. 18, no. 16, pp. 3774-3779, 2006.

[102] J. Chen, W.-S. Liao, X. Chen et al., "Evaporation-induced assembly of quantum dots into nanorings," ACS Nano, vol. 3, no. 1, pp. 173-180, 2009.

[103] Y. Cai and B. M. Ocko, "Large-scale fabrication of protein nanoarrays based on nanosphere lithography," Langmuir, vol. 21, no. 20, pp. 9274-9279, 2005.

[104] Q. Yu, H. Huang, R. Chen, H. Yang, X. Peng, and Z. Ye, "Filtration-assembling colloidal crystal templates for ordered macroporous nanoparticle films," Journal of Materials Chemistry, vol. 21, no. 44, pp. 18089-18094, 2011.

[105] X. Wang, C. J. Summers, and Z. L. Wang, "Large-scale hexagonal-patterned growth of aligned $\mathrm{ZnO}$ nanorods for nano-optoelectronics and nanosensor arrays," Nano Letters, vol. 4, no. 3, pp. 423-426, 2004.

[106] Y. Li, T. Sasaki, Y. Shimizu, and N. Koshizaki, "Hexagonal-closepacked, hierarchical amorphous $\mathrm{TiO}_{2}$ nanocolumn arrays: transferability, enhanced photocatalytic activity, and superamphiphilicity without UV irradiation," Journal of the American Chemical Society, vol. 130, no. 44, pp. 14755-14762, 2008.

[107] L. Li, Y. Li, S. Gao, and N. Koshizaki, “Ordered $\mathrm{Co}_{3} \mathrm{O}_{4}$ hierarchical nanorod arrays: tunable superhydrophilicity without UV irradiation and transition to superhydrophobicity," Journal of Materials Chemistry, vol. 19, no. 44, pp. 8366-8371, 2009.

[108] Y. Li, N. Koshizaki, Y. Shimizu, L. Li, S. Gao, and T. Sasaki, "Unconventional lithography for hierarchical micro/nanostructure arrays with well-aligned 1D crystalline nanostructures: design and creation based on the colloidal monolayer," ACS Applied Materials and Interfaces, vol. 1, no. 11, pp. 2580-2585, 2009.

[109] P. Colson, A. Schrijnemakers, B. Vertruyen, C. Henrist, and R. Cloots, "Nanosphere lithography and hydrothermal growth: how to increase the surface area and control reversible wetting properties of $\mathrm{ZnO}$ nanowire arrays?" Journal of Materials Chemistry, vol. 22, no. 33, pp. 17086-17093, 2012.

[110] Y. Li, J. Zhang, S. Zhu et al., "Bioinspired silicon hollowtip arrays for high performance broadband anti-reflective and water-repellent coatings," Journal of Materials Chemistry, vol. 19, no. 13, pp. 1806-1810, 2009.

[111] Z. Huang, H. Fang, and J. Zhu, "Fabrication of silicon nanowire arrays with controlled diameter, length, and density," Advanced Materials, vol. 19, no. 5, pp. 744-748, 2007.

[112] W. Li, L. Xu, W.-M. Zhao, P. Sun, X.-F. Huang, and K.-J. Chen, "Fabrication of large-scale periodic silicon nanopillar arrays for 2D nanomold using modified nanosphere lithography," Applied Surface Science, vol. 253, no. 22, pp. 9035-9038, 2007.

[113] H. L. Chen, S. Y. Chuang, C. H. Lin, and Y. H. Lin, "Using colloidal lithography to fabricate and optimize sub-wavelength pyramidal and honeycomb structures in solar cells," Optics Express, vol. 15, no. 22, pp. 14793-14803, 2007.

[114] B. Wang, W. Zhao, A. Chen, and S.-J. Chua, "Formation of nanoimprinting mould through use of nanosphere lithography," Journal of Crystal Growth, vol. 288, no. 1, pp. 200-204, 2006.
[115] B. Varghese, F. C. Cheong, S. Sindhu et al., "Size selective assembly of colloidal particles on a template by directed selfassembly technique," Langmuir, vol. 22, no. 19, pp. 8248-8252, 2006.

[116] Y. Yin, Y. Lu, B. Gates, and Y. Xia, “Template-assisted selfassembly: a practical route to complex aggregates of monodispersed colloids with well-defined sizes, shapes, and structures," Journal of the American Chemical Society, vol. 123, no. 36, pp. 8718-8729, 2001.

[117] A. Kosiorek, W. Kandulski, H. Glaczynska, and M. Giersig, "Fabrication of nanoscale rings, dots, and rods by combining shadow nanosphere lithography and annealed polystyrene nanosphere masks," Small, vol. 1, no. 4, pp. 439-444, 2005.

[118] G. Zhang and D. Wang, "Fabrication of heterogeneous binary arrays of nanoparticles via colloidal lithography," Journal of the American Chemical Society, vol. 130, no. 17, pp. 5616-5617, 2008.

[119] Y. Jinling, D. Guotao, and C. Weiping, "Controllable fabrication and tunable magnetism of nickel nanostructured ordered porous arrays," Journal of Physical Chemistry C, vol. 113, no. 10, pp. 3973-3977, 2009.

[120] S. H. Lee, K. C. Bantz, N. C. Lindquist, S.-H. Oh, and C. L. Haynes, "Self-assembled plasmonic nanohole arrays," Langmuir, vol. 25, no. 23, pp. 13685-13693, 2009.

[121] K. Kempa, B. Kimball, J. Rybczynski et al., "Photonic crystals based on periodic arrays of aligned carbon nanotubes," Nano Letters, vol. 3, no. 1, pp. 13-18, 2003.

[122] Y. Li, T. Sasaki, Y. Shimizu, and N. Koshizaki, "Hexagonal-closepacked, hierarchical amorphous $\mathrm{TiO}_{2}$ nanocolumn arrays: transferability, enhanced photocatalytic activity, and superamphiphilicity without UV irradiation," Journal of the American Chemical Society, vol. 130, no. 44, pp. 14755-14762, 2008.

[123] Y. Li, X. Fang, N. Koshizaki et al., "Periodic $\mathrm{TiO}_{2}$ nanorod arrays with hexagonal nonclose-packed arrangements: excellent field emitters by parameter optimization," Advanced Functional Materials, vol. 19, no. 15, pp. 2467-2473, 2009.

[124] C.-W. Kuo, J.-Y. Shiu, Y.-H. Cho, and P. Chen, "Fabrication of large-area periodic nanopillar arrays for nanoimprint lithography using polymer colloid masks," Advanced Materials, vol. 15, no. 13, pp. 1065-1068, 2003. 

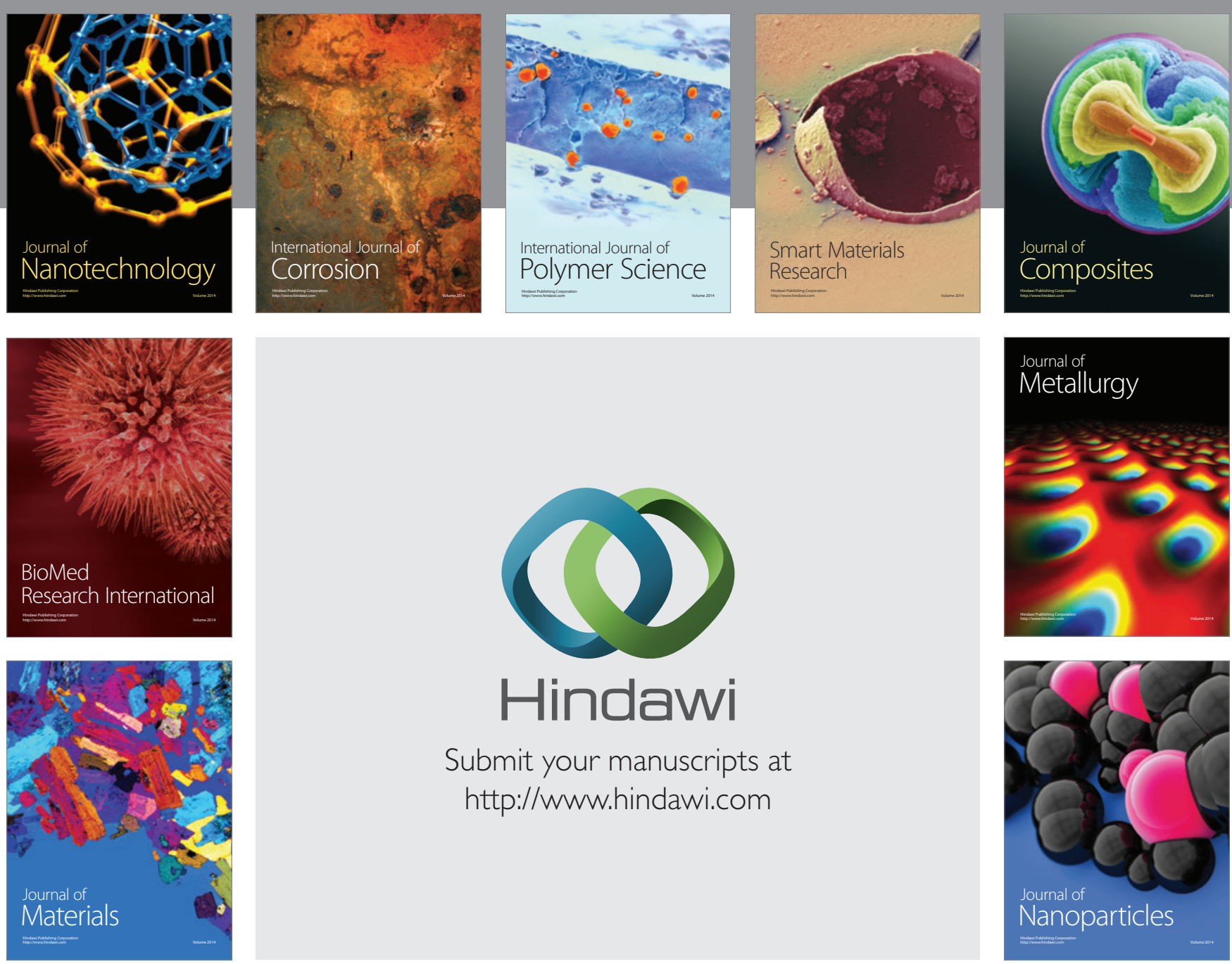

Submit your manuscripts at http://www.hindawi.com
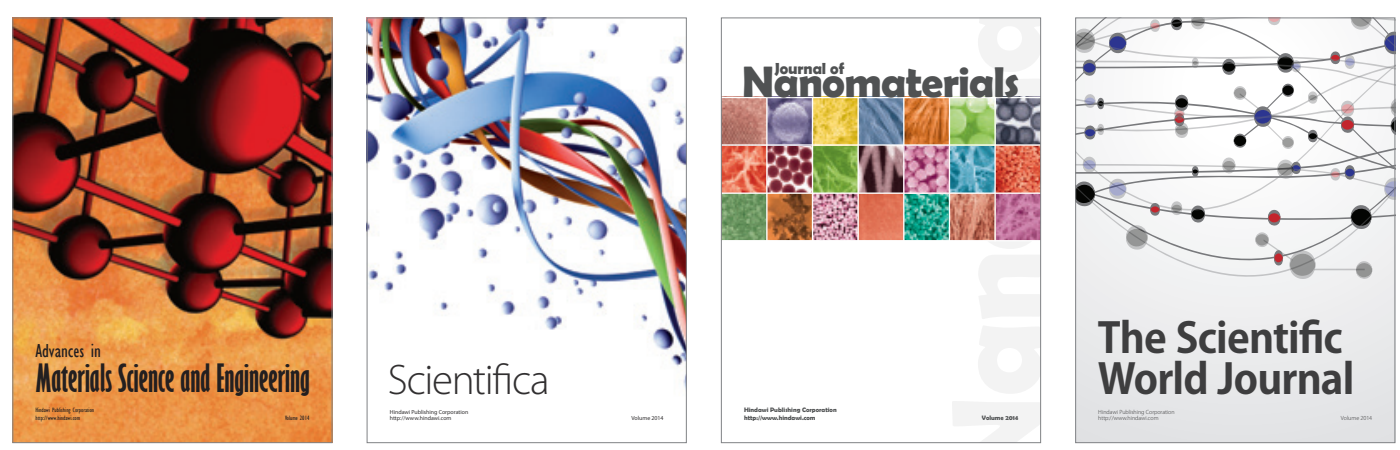

\section{The Scientific World Journal}
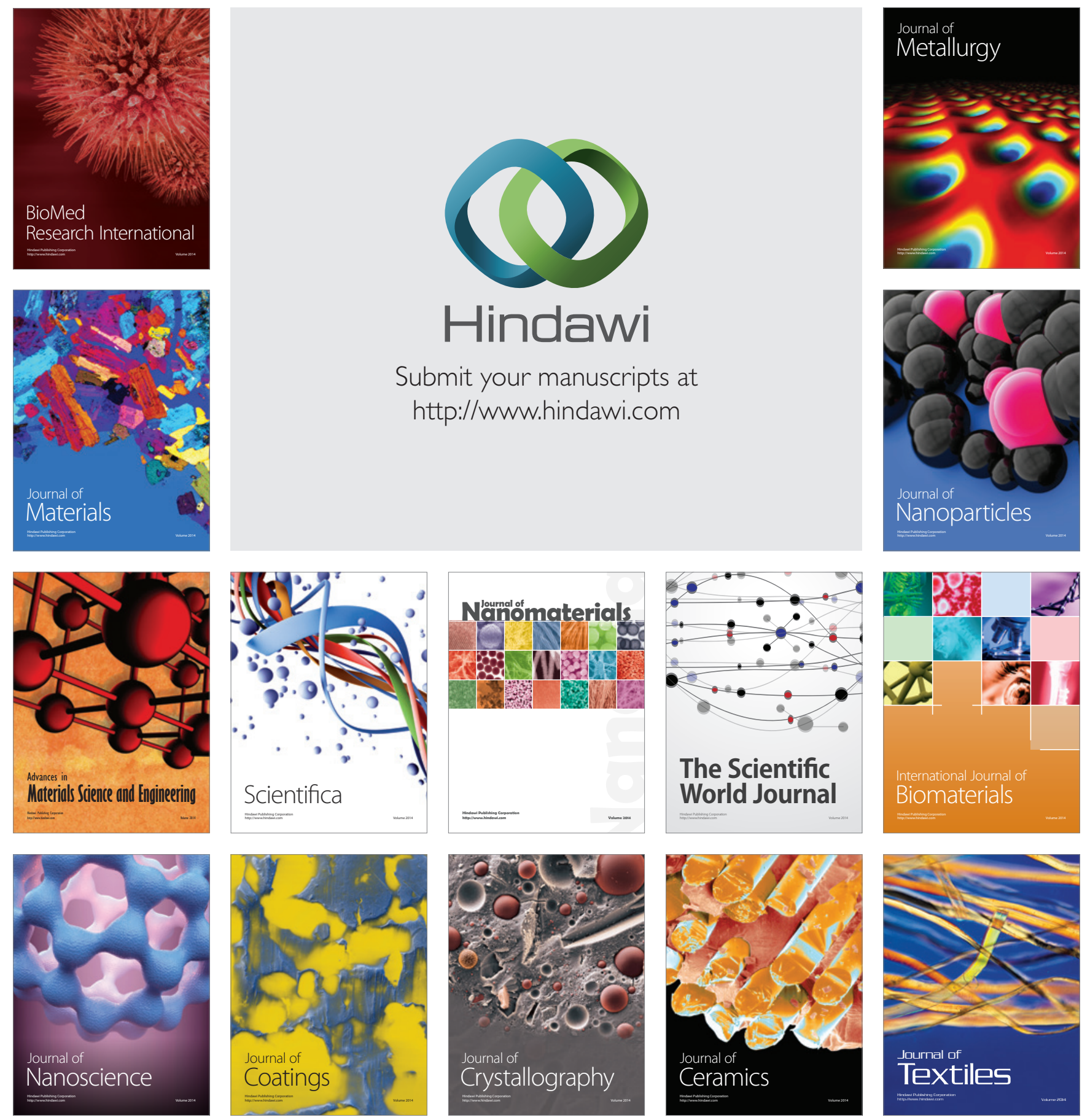